\title{
Digitale Disruption und Transformation. Herausforderungen für Recht und Rechtswissenschaft
}

\author{
Wolfgang Hoffmann-Riem
}

„Das Alte stirbt, es ändert sich die Zeit, ein neues Leben blübt aus den Ruinen" (Friedrich Schiller, „Wilhelm Tell“, 4. Akt, 2. Szene).

Meinen herzlichen Dank an die Bucerius Law School für dieses aus Anlass meines 80. Geburtstags durchgeführte Symposium, an Martin Eifert als Ideengeber für das Gesamtthema und als wissenschaftlicher Koordinator, an die Referenten für deren vorzügliche Referate sowie an die sonstigen Teilnehmer/innen für deren wertvolle Anregungen kann ich hier nur allgemein, möchte ibn aber dennoch mit Nachdruck aussprechen. Ich füge hinzu, dass es für mich als Wissenschaftler ein besonderes Geschenk ist, durch weiterführende Referate zu einem mich inhaltlich bewegenden Thema geehrt zu werden. Das Programm sah ein Schlusswort von mir vor, in dem ich meinen Dank auszudrücken versuchte, aber zusätzlich - wenn auch notgedrungen knapp - inhaltliche Anmerkungen formulieren konnte. Der folgende Text ist nicht auf diese Anmerkungen begrenzt, sondern setzt Gedanken zu dem Thema Digitalisierung fort, die mich seit längerem beschäftigen.

\section{Wandel, Umbrüche, Transformationen}

\section{Zur Begrifflichkeit}

Der im Titel dieses Beitrags gewählte Begriff Disruption verweist einerseits auf Prozesse der Zerstörung von Gewissheiten und der Abkehr von Gewohnheiten, aber auch auf Wege des Aufbruchs zu Neuem, etwa die Neuschaffung von Strukturen oder die Einleitung gravierender Wandlungsprozesse. Führt dies zu dauerhaften, insbesondere fundamentalen, Veränderungen etwa von Institutionen, von Denkweisen oder von technologischen Möglichkeiten sowie zu veränderten Produkten, Dienstleistungen oder andersartigen erheblichen Auswirkungen auf Chancen und Risiken der individuellen und gesellschaftlichen Lebensgestaltung, wird häufig der Begriff Transformation verwendet. Die im Änderungsprozess genutzten neuen Technologien werden den „transformativen Technologien“ zugeordnet. 
Die Redeweise von der digitalen Transformation bezieht sich übergreifend auf den durch die Digitalisierung bewirkten Veränderungsprozess. Ausgangspunkt sind technologische Innovationen. Umfasst von der digitalen Transformation sind u. a. durch die Digitalisierung ausgelöste neue Wege zu Wissen, Veränderungen der Kommunikation, damit verbunden neue Handlungsmöglichkeiten und Geschäftsmodelle. Nachhaltige Änderungen lassen sich in Wirtschaft, Gesellschaft, Politik, Recht u.v.a.m. beobachten.

\section{Insbesondere: Die digitale Transformation ${ }^{1}$}

Aus technologischer Sicht sind für die digitale Transformation besonders wichtig: Algorithmische Systeme, Big Data Analytics, künstliche Intelligenz (KI) und in deren Rahmen neuronale Netze, das Internet der Dinge (IoT), Blockchain und Robotik sowie die für digitale Techniken und deren Vernetzung eingerichteten Infrastrukturen. Solche technologisch fundierten Neuerungen ermöglichen Veränderungen in verschiedenen Bereichen, so den Aufbau sozialer Netzwerke (etwa Facebook) und anderer Kommunikationsdienste (etwa Instant Messaging) und Kommunikationsformen (etwa Social Bots) ${ }^{2}$, Veränderungen der gewohnten Lebensgestaltung (etwa im Smart Home), aber auch die Schaffung neuer Überwachungssyste-

1 Speziell zur digitalen Transformation s. L. Floridi, Die 4. Revolution - Wie die Infosphäre unser Leben verändert, 2015; A. Bounfour, Digital Futures, Digital Transformation, 2016; F. Stalder, Kultur der Digitalität, 2016; T. Cole, Digitale Transformation, 2. Auflage 2017; C. Keese, Silicon Germany: Wie wir die digitale Transformation schaffen, 2017; O. Stengel/A. van Looy/S. Wallaschkowski (Hrsg.), Digitalzeitalter - Digitalgesellschaft: Das Ende des Industriezeitalters und der Beginn einer neuen Epoche, 2017; R. Pfliegl/C. Seibt, Die digitale Transformation findet statt!, e\&i Elektrotechnik und Informationstechnik, 2017, 333; V. Mayer-Schönberger/T. Ramge, Das Digital. Markt, Wertschöpfung und Gerechtigkeit im Datenkapitalismus, 2017; A. Rolf, Weltmacht Vereinigte Daten. Die Invasion des Digitalen verstehen, 2018; B. Kolany-Raiser/R. Heil/C. Orwat/T. Hoeren (Hrsg.), Big Data und Gesellschaft: Eine multidisziplinäre Annäherung, 2018; H. Zech, Künstliche Intelligenz und Haftungsfragen, ZfPW 2019, 198; S. Zuboff, Das Zeitalter des Überwachungskapitalismus, 2018; A. Nassehi, Muster. Theorie der digitalen Gesellschaft, 2019.

2 Social Bots sind von Menschen programmierte Computerprogramme, die eine menschliche Identität vortäuschen und zu manipulativen Zwecken eingesetzt werden, indem sie wie Menschen im Internet agieren, s. M. Libertus, Rechtliche Aspekte des Einsatzes von Social Bots de lege lata und de lege ferenda, ZUM 2016, 20. 
me zur Nutzung sowohl durch private als auch durch hoheitliche Stellen u.v.a.m. ${ }^{3}$

Dabei wäre es verfehlt, die Nutzungen digitaler Techniken - wie es häufig vor allem in der allgemeinen Öffentlichkeit geschieht - in erster Linie unter dem Aspekt des personenbezogenen Datenschutzes und der Beeinflussung der Meinungsbildung in der Gesellschaft zu thematisieren. Digitale Techniken greifen weit darüber hinaus. Erwähnt sei bespielhaft der Einsatz cyberphysischer Systeme für neuartige vernetzte und automatisierte und zum Teil autonomisierte Produktionsprozesse ("Industrie 4.0"), für selbstfahrende Fahrzeuge, aber auch für die Sicherung der Leistungsfähigkeit von Infrastrukturen der Mobilität oder der Energieversorgung oder auch für die hoheitliche Überwachung. Darüber hinaus werden digitale Techniken in fast allen Lebensbereichen eingesetzt und der Zugriff auf digitale Techniken - etwa über die Nutzung des Internet, des Smartphone oder von Rundfunk und Fernsehen - prägt das tägliche Leben fast aller Menschen. Daher liegt es nicht fern, von einer tiefgreifenden „strukturellen Transformation der Gesellschaft" zu sprechen. ${ }^{4}$

Die vielfältigen Verwendungsmöglichkeiten können sich nicht mehr allein auf die deterministisch mit Hilfe vorprogrammierter Entscheidungsschritte arbeitenden algorithmischen Systeme stützen. Immer wichtiger werden lernende Algorithmen, die insbesondere unter Nutzung künstlicher Intelligenz ${ }^{5}$ nicht oder doch nicht ausschließlich nach einem definierten Muster und in der Folge mit Hilfe vorgegebener Entscheidungsschritte

3 E. Peuker, Verfassungswandel durch Digitalisierung. Digitale Souveränität als verfassungsrechtliches Leitbild, 2020 kennzeichnet in These 1 der Zusammenfassung als Digitalisierung in einem weiten Sinne: die Dynamik der technischen Entwicklung, die Vernetzung der digitalen Endgeräte, die Konvergenz von Infrastrukturen, Endgeräten und Diensten, die Ubiquität der Informations- und Kommunikationstechnik sowie die Datafizierung.

4 So etwa M. Oermann, Gewährleistung der Möglichkeit internetbasierter Kommunikation, 2018, 22.

$5 \mathrm{Zu}$ ihr s. allg. S. Russell/P. Norvig, Künstliche Intelligenz, 3. Aufl. 2012; Ertel, Grundkurs künstliche Intelligenz, 4. Aufl. 2016; bitkom, Künstliche Intelligenz verstehen als Automation des Entscheidens, Leitfaden 2017. Zu Einsatzmöglichkeiten s. Die Bundesregierung, Eckpunkte der Bundesregierung für eine Strategie Künstliche Intelligenz vom 18. Juli 2018; Unabhängige Expertengruppe für künstliche Intelligenz, Ethik-Leitlinien für eine vertrauenswürdige KI, 2019 - eingesetzt von der EUKommission - , gibt im Glossar (S.49) eine ausführliche Definition von KI.; $H$. Zech (Fn. 1); M. Martini, Blackbox Algorithmus - Grundfragen einer Regulierung Künstlicher Intelligenz, 2019, $19 \mathrm{ff}$; W. Hoffmann-Riem, Artificial intelligence as a Challenge for Law and Regulation, in: T. Wischmeyer/T. Rademacher (Hrsg.), Regulating Artificial Intelligence, 2020, 1 ff.; Europäische Kommission, Weißbuch. Zur 
vorgehen, um Eingaben des Anwenders zu verarbeiten. Vielmehr können sie unter Rückgriff auf eigene Wahrnehmungen und frühere Erfahrungen vorgehen. ${ }^{6}$ Sie können insbesondere über die Fähigkeit verfügen, Muster zu erkennen und gelernte Muster auf neue Datensätze anzuwenden. Sogenannte maschinelle Lernsysteme ${ }^{7}$ haben $u$. a. die Fähigkeit, Bilder zu bewerten, Sprache in Texte zu übersetzen oder Prognosen auf der Grundlage der Auswertung von Vergangenem zu erstellen. Besonders hoch entwickelte intelligente Systeme können Zusammenhänge, Strukturen und Architekturen so erfassen, dass sie in der Lage sind, sich neuen Problemsituationen anzupassen, indem sie ihre Entscheidungsregeln autonom, also unabhängig von der menschlichen Programmierung, fortentwickeln. Möglich ist es auch, dass die Programme sich unter Nutzung neuronaler Netze ${ }^{8}$ durch Schaffung neuer Vernetzungen zwischen den Neuronen fortentwickeln. Hier wird von "Deep Learning" gesprochen.?

\section{Anpassungsdruck durch aktuelle Ereignisse: Die Corona-Pandemie}

Die Digitalisierung ist durch starke Dynamiken geprägt, gekoppelt mit der Möglichkeit, flexibel auf neue Problemsituationen zu reagieren. So wird mit hoher Wahrscheinlichkeit die im Jahre 2020 ausgebrochene CoronaPandemie zu spezifischen, aber vermutlich dauerhaft fortwirkenden Transformationsfolgen führen, dies auch hinsichtlich der Einsatzbereiche der digitalen Techniken, aber auch der durch ihre Nutzung speziell für die Pandemiebekämpfung bewirkten Veränderungen von Lebensgewohnheiten.

Künstlichen Intelligenz - ein europäisches Konzept für Exzellenz und Vertrauen, 19.02.2020, COM (2020), 65 final.; M. Ebers/ C. Heinze/T. Krügel/B. Steinrötter (Hrsg.). Künstliche Intelligenz und Robotik. Rechtshandbuch, 2020 (i. E.).

6 Nähere Erklärungen bei H. Zech, (2020). Risiken Digitaler Systeme: Robotik, Lernfähigkeit und Vernetzung als aktuelle Herausforderungen für das Recht. (Weizenbaum Series, 2), https://doi.org/10.34669/wi.ws/2.

7 Zum Machine Learning und dessen Anwendung beim juristischen Arbeiten: $H$. Surden, Machine Learning and Law, 89 Washington Law Review 2014, 87 ff.; E. Alpaydin, Machine Learning, 2016. Das Wort Maschine (machine) ist hier als Rechenregel oder Algorithmus zu verstehen.

8 Zu ihnen s. H. Zech (Fn. 1), $201 \mathrm{f}$.

$9 \mathrm{Zu}$ ihm s. I. Goodfellow/Y. Bengio/A. Courville, Deep Learning, 2016; F. Stalder (Fn. 1), 177 ff.; C. D. Müller-Hengstenberg/S. Kirn, Intelligente "Software-Agenten": Eine neue Herausforderung für unser Rechtssystem? Multimedia \& Recht 2014, 307 ff.; M. Martini (Fn. 5), 23 f. 
Bei der Analyse ihrer Verläufe und bei der Bewältigung der Probleme der Pandemie wurden und werden verstärkt digitale Technologien unter Einschluss lernender Systeme eingesetzt. ${ }^{10}$ Dies betrifft zunächst insbesondere den Einsatz in der Epidemiologie und Virologie, darunter die Analyse und Prognose des Ablaufs der Pandemie und die Entwicklung von Testverfahren. Es gilt ferner für die Entwicklung von Medikamenten zur Immunstärkung und zur Heilung. Anstöße zu transformativen Veränderungen werden vermutlich auch die Erfahrungen mit der Nutzung digitaler Technologien und mit den gesellschaftlichen Begleitfolgen des Umgangs mit der Pandemie geben. Zu erwähnen sind die angesichts von Ausgangssperren vermehrte Arbeit im Homeoffice, das für die Dauer der Schulschließungen genutzte Homeschooling, der digitale Fernunterricht in Hochschulen, Online-Meetings wie Videokonferenzen oder Webinare sowie andere genutzte Möglichkeiten der elektronisch gestützten Zusammenarbeit an unterschiedlichen Örtlichkeiten tätiger Personen. Diese Vorgehensweisen werden voraussichtlich nachhaltige Auswirkungen auf das Bildungssystem, die Arbeitsbedingungen und den Arbeitsmarkt haben, aber auch auf die Art der weltweiten Zusammenarbeit im Bereich der Wirtschaft, so bei der Produktion und der Gestaltung von Lieferketten.

\section{Historische Beispiele von Transformationen}

Im Laufe der Geschichte haben Gesellschaften transformative Umbrüche unterschiedlicher Art erlebt. Solche Umbrüche waren häufig mit einer veränderten Wahrnehmung und Deutung des Weltgeschehens verbunden. Als Beispiel für ein solches Umdenken sei die Kopernikanische Wende erwähnt, also die Abkehr von einem geozentrischen hin zu einem heliozentrischen Weltbild und in der Folge die Korrektur vieler zuvor als unveränderlich angenommenen Vorstellungen insbesondere in Philosophie und Religion.

Besonders nachhaltig waren aber auch technische Entdeckungen, die neue Problemlösungen ermöglichten. Ein Beispiel dafür und für damit verbundene gesellschaftliche und politische Neuerungen ist die Erfindung des Buchdrucks. Sie beschränkte vorherige Exklusivitäten des Informati-

10 So ist beispielsweise Machine Learning im Umgang mit der Corona-Pandemie für folgende Zwecke eingesetzt worden: Um Risikogruppen zu identifizieren, Patienten zu diagnostizieren, Medikamente schneller zu entwickeln, die Ausbreitung des Virus vorherzusagen, Viren besser zu verstehen, und um festzustellen, woher ein Virus kommt. 
onszugangs, eröffnete ungeahnte Lern- und Bildungsmöglichkeiten auch für Massen und erleichterte beispielsweise neue soziale Bewegungen. Weitere Beispiele sind die Erfindung der Dampfmaschine und die damit verkoppelte Industrialisierung. Erwähnt seien ferner Veränderungen in der Art der Kriegsführung, aber auch Umwälzungen von Kulturen, etwa aus Anlass von Migrationsbewegungen. Immer wieder waren auch spezielle neue Technologien Anstöße für Wandel, so die Atomenergie, die Bio- und Gentechnologie, die Nanotechnologie, die Medizintechnologie, aber auch die Informationstechnologien unter Einschluss der aktuellen Veränderungen durch die Digitalisierung.

Anstöße zu Umbrüchen gaben nicht nur veränderte Sichtweisen und darauf gegründetes neues Wissen - wie bei der Kopernikanischen Wende -, sondern auch Krisen, zurzeit etwa die Klimakrise und die durch die schon erwähnte Corona-Pandemie ausgelösten wirtschaftlichen, politischen und sozialen Funktionsstörungen in vielen gesellschaftlichen Bereichen, so u. a. im Gesundheitssektor, in der Wirtschaft und in der Politik.

Prozesse des Zusammenspiels zwischen Disruption und Transformation führen zu Herausforderungen auch für die Wissenschaft, nicht nur bei der Analyse von konkreten Erscheinungen, sondern auch bei der Suche nach theoretischen Erklärungen und angemessenen methodischen Vorgehensweisen. Das hat Thomas Kubn am Beispiel der Bedeutung des Wechsels von Paradigmen - also der Aufgabe bisher leitender Denkrahmen - beschrieben. ${ }^{11}$ Ähnlich aufschlussreich sind Ludwig Flecks Arbeiten zur Bedeutung von veränderten Denkstilen für neue Entwicklungen ${ }^{12}$ sowie die seit einiger Zeit erfolgende konstruktivistische Wende in weiten Teilen der Sozial-, Technik- und Rechtswissenschaft. ${ }^{13}$ Die Betonung des transformativen

11 T. S. Kuhn, Die Struktur wissenschaftlicher Revolutionen, 1989 (Org. 1962).

12 L. Fleck, Entstehung und Entwicklung einer wissenschaftlichen Tatsache. Einführung in die Lehre vom Denkstil und Denkkollektiv, 1935.

13 S. statt vieler $P$. Watzlawick, Erfundene Wirklichkeit - Wie wissen wir, was wir zu wissen glauben? Beiträge zum Konstruktivismus, 1981; P. L. Berger/T. Luckmann, Die gesellschaftliche Konstruktion der Wirklichkeit, 1969; H. von Foerster/E. von Glasersfeld/P. M. Hejl/S. J. Schmidt, P. Watzlawick, Einführung in den Konstruktivismus, 1992; J. Halfmann, Wissenschaft, Methode und Technik - Die Geltungsüberprüfung von wissenschaftlichem Wissen durch Technik, in: C. Engel/J. Halfmann/M. Schulte (Hrsg.), Wissen, Nichtwissen, unsicheres Wissen. Disziplinäre und interdisziplinäre Annäherungen, 2002, 227; B. Pörksen, Schlüsselwerke des Konstruktivismus, 2014; $R$. Martinsen, Spurensuche: Konstruktivistische Theorien der Politik, 2014. Aus politikwissenschaftlicher Sicht s. etwa A. Wendt, Social Theory of International Politics, 1999. Aus juristischer Sicht siehe K. I. Lee, Die 
Charakters lässt sich auch als eine spezifische Rahmung ${ }^{14}$ der Diskurse über die Digitalisierung, deren Bewertung und mögliche Konsequenzen in der Rechtsordnung verstehen.

\section{Insbesondere: Zu Einordnungen von Disruptionen der Wirtschaftsordnung}

In diesem Beitrag sollen Wirkungen der durch die Digitalisierung ausgelösten Disruptionen und Transformationen in Gestalt von Reaktionen im Recht und in der Rechtwissenschaft im Zentrum stehen. Zunächst aber soll die Bedeutung ökonomischer Veränderungen und deren Wahrnehmung thematisiert werden, denn gerade das Zusammenspiel zwischen den neuen digitalen Technologien und ihrer Nutzung in wirtschaftlichen Aktivitäten prägt in einem erheblichen Maße auch die Möglichkeit und Notwendigkeit rechtlicher Reaktion.

Die Nutzung des Begriffs der Disruption zur Kennzeichnung der eingangs erwähnten Wandlungsprozesse ist relativ neu. Er kann aber rückbezüglich auch auf vergangene Erscheinungen angewandt werden. So steht die Idee disruptiv wirkender Änderungsprozesse hinter der von Karl Marx analysierten Verdrängung alter Ordnungen - bei ihm des Feudalismus und der für diesen typischen Produktionsweise - und deren Transformation in eine neue, nämlich die kapitalistische, Ordnung. Dies wurde bei Marx zum zentralen Gegenstand fundamentaler Kritik. ${ }^{15}$

Karl Polanyi hat in seiner Beschreibung der "Großen Transformation"16 am Beispiel Englands Folgen der Ablösung der Agrarwirtschaft durch die industrielle Revolution beschrieben. Dies war ihm Anlass für die analytische Durchdringung des transformativen Prozesses der Durchsetzung eines selbstregulativen Marktes und der Kommerzialisierung von Land, Kapital und Arbeit sowie in der Folge der Verselbständigung der Wirtschaft

Struktur der juristischen Entscheidung aus konstruktivistischer Sicht, 2010; N. Just/M. Latzer, Governance by Algorithms: Reality Construction by Algorithmic Selection on the Internet, in: Media, Culture \& Society, 2016, 1 ff.;I. Schulz-Schaeffer, Innovation als soziale Konstruktion von Technik und Techniknutzung, in: B. Blättel-Mink/I. Schulz-Schaeffer/A. Windeler (Hrsg.), Handbuch Innovationsforschung, 2019.

14 In Bezug genommen ist damit der Framingansatz. Zu ihm s. C. Jecker, Entmans Framing-Ansatz, 2017; J. Matthes, Framing, 2. Aufl. 2020.

15 K. Marx, Das Kapital, Kritik der politischen Ökonomie, in: K. Marx/F. Engels, Marx-Engels-Werke, Bd. 23-25, Neuausgabe 1973.

16 K. Polanyi, The Great Transformation. The Political and Economic Origins of Our Time, 2001 (Org. 1944). Zu den Ausführungen im Text s. insbesondere 35 ff. 
gegenüber der Gesellschaft. Als zerstörerische Wirkung hat er die damit verbundene Transformation der natürlichen und menschlichen Substanz der Gesellschaft in Waren und die Durchsetzung des Utilitarismus angesehen.

Josef Schumpeter hat unter Rückgriff auf die (schon ältere) Metapher der schöpferischen Zerstörung in der kapitalistischen Ordnung demgegenüber besonders günstige Voraussetzungen insbesondere für Innovationen - etwa technisch-ökonomisch fortschrittliche Innovationen - und damit verbundene Möglichkeiten zur Steigerung des Wohlstands gesehen. ${ }^{17}$

In jüngerer Zeit hat Clayton Christensen den Begriff der Disruption mit einer spezifischen Blickrichtung, nämlich der auf marktverdrängende Prozesse, in der Wissenschaft populär gemacht. Er verwendet den Disruptionsbegriff nicht allgemein für die Beschreibung von Anlässen und Folgen von bahnbrechenden, etwa technologischen Innovationen. Sein Thema sind vorrangig marktbezogene Prozesse, insbesondere solche, durch die ein kleines Unternehmen mit geringen Ressourcen es schafft, mit neuen erfolgreichen Produkten ein etabliertes Unternehmen vom Markt zu verdrängen. In das Umfeld der Disruption ordnet er es auch, wenn bisher erfolgreiche Unternehmen diese neuen Leistungen adaptieren und selbst zu erbringen suchen. ${ }^{18}$

Im Zentrum der Theorie von Clayton steht die Entwicklung neuer Möglichkeiten am Markt und die Zurückdrängung etablierter Anbieter. Besonders wichtig erscheint es mir aber auch, eine Gegenentwicklung zu benennen, nämlich die insbesondere mit der Globalisierung wesentlicher Teilmärkte im IT-Bereich verbundenen Veränderungen von Marktstrukturen im Sinne der Herausbildung von oligopolistischer oder gar monopolistisch ausgestalteter Macht, ${ }^{19}$ und zwar gegenwärtig ohne erkennbare Aussicht auf strukturelle Bändigung. Zur Erklärung dieses Phänomens verweist die

17 J. A. Schumpeter, Theorie der wirtschaftlichen Entwicklung, 1989 (Org. 1962).

$18 \mathrm{Zu}$ dem zugrunde gelegten Disruptionsverständnis s. C. M. Christensen/M. E. Raynor/R. MacDonald, What Is Disruptive Innovation?, Harvard Business Review, Dezember 2015, https://hbr.org/2015/12/what-is-disruptive-innovation.

$19 \mathrm{Zu}$ Fragen der Marktmacht und die Möglichkeit ihrer rechtlichen Einhegung s. H. Schweitzer/M. Peitz, Datenmärkte in der digitalisierten Wirtschaft: Funktionsdefizite und Regelungsbedarf? ZEW Discussion Paper, http://ftp.zew.de/pub/zewdocs/dp/dp17043.pdf.; M. Volmar, Digitale Marktmacht, 2019 m. w. Hinw. S.; M. Martini (Fn. 59), 62 ff, 103 ff. S. auch den von der EU-Kommission in Auftrag gegebenen Report: J. Crémer/Y-A. de Montoye/H. Schweitzer, Competion policy for the digital era, 2019. 
Internet- bzw. Informationsökonomie ${ }^{20}$ insbesondere auf die mit Informationsgütern und ihren Märkten verbundenen spezifischen Eigenschaften. Ich nenne nur Stichworte: Die Nichtrivalität im Konsum solcher Güter, ferner der Umstand, dass selbst bei hohen Fixkosten ihrer Erstellung die Durchschnittskosten der Informationserzeugung und -vervielfältigung unendlich fallen, da nur geringe variable Kosten entstehen und die Güter sich beim Konsum praktisch nicht verbrauchen. Wichtig ist ferner die Mehrseitigkeit der Märkte. Hinzu treten Netzwerkeffekte: Die Informationsgüter haben für die Unternehmen, aber vielfach auch für die Konsumenten einen umso höheren Nutzen, je größer die Zahl derjenigen ist, die bereits mit dem Netz verbunden sind und es nutzen. $\mathrm{Zu}$ solchen direkten Netzeffekten kommen indirekte Netzeffekte, nämlich die Nutzung der Daten auch in anderen Geschäftsbereichen der Unternehmen und durch deren Weitergabe zur Nutzung durch Dritte, etwa an die Unternehmen der Werbewirtschaft. Netzeffekte ermöglichen angesichts der (Re)Kombinierbarkeit von Daten unterschiedlicher Art und Herkunft sowie ihrer Offenheit zur Nutzung für vielfältige Zwecke die Schaffung erheblichen Mehrwerts.

Die durch diese Eigenschaften von Informationsgütern und die neuen Strukturen digitaler Märkte ermöglichten hohen Erträge führen zur Verfügbarkeit von erheblichen Finanzmitteln, die den erfolgreichen Unternehmen beispielsweise den Aufkauf von innovativen Start-ups bzw. der von diesen entwickelten Ideen und deren Nutzung zur Herstellung von Produkten und Diensten ermöglichen. ${ }^{21}$ Ferner werden Allianzen mit marktstarken Unternehmen geschlossen, auch mit solchen, die in anderen, etwa industriellen, Tätigkeitsfeldern arbeiten. Derartige Vorgehensweisen erlauben es den Marktstarken, ihre Marktstellung zu verteidigen und zu stärken, selbst wenn ihre eigene Innovationskraft schon ermüdet ist.

$20 \mathrm{Zu}$ ihr s. statt vieler R. Peters, Internet-Ökonomie, 2010; R. Clement/D. Schreiber, Internet-Ökonomie: Grundlagen und Fallbeispiele der vernetzten Wirtschaft, 2016.

21 Plastisch dazu A. Rolf/A. Sagawe, Des Googles Kern und andere Spinnennetze, 2015. 


\section{Aktuelle Deutungen der digitalen Disruption und Transformation}

\section{Ausforschungskapitalismus}

Die US-amerikanische Wirtschaftswissenschaftlerin Shoshana Zuboff hat einen spezifischen Erklärungsansatz für die Machtakkumulation und damit verbundene Disruptionen formuliert und mit einem provozierenden Begriff versehen: Überwachungskapitalismus („Surveillance Capitalism"). ${ }^{22}$ Dieser schere aus der Geschichte des Marktkapitalismus aus. ${ }^{23}$ Zentral ist ihre Annahme, dass die digitalen Informationstechnologien dazu taugen und eingesetzt werden, in großem Stil Verhaltensdaten der Nutzer der Dienste $-\mathrm{m}$. E. besser: Informationen ${ }^{24}$ über das Verhalten von Nutzern - zu erheben. Diese Informationen sind für die Erbringung und Verbesserung von Produkten und Dienstleistungen für die Nutzer hilfreich und werden dafür auch eingesetzt. Sie dienen aber ebenfalls und vor allem zur Schaffung oder Verbesserung von eigenständig einsetzbaren wertvollen Produktionsmitteln. Zuboff nennt dieses Mehr einen Verhaltensüberschuss (Behavioral Surplus). ${ }^{25}$ Gewonnen werde er aus Informationen über das Online-Verhalten der Nutzer (Browsing, Suche, Social Media), indem jede Bewegung, jedes Gespräch, jeder Gesichtsausdruck, jeder Laut, jeder Text, jedes Bild, das der ubiquitären Extraktionsarchitektur zugänglich ist, festgehalten werden kann und vielfach auch wird. Die Menschen, deren Verhaltensweisen erfasst und dokumentiert werden, seien eine kostenlose Quelle für den dadurch entstehenden Rohstoff, der zur Herstellung marktfähiger und häufig hoch lukrativer Produkte genutzt werde.

Mir scheint allerdings der Begriff „Surveillance/Überwachung“ für dieses Vorgehen insofern nicht optimal, als dieser jedenfalls in Deutschland meist eng mit der Vorstellung der Überwachung einzelner Personen oder Abläufe als mögliche Grundlage für gegen diese gerichtete Maßnahmen eingesetzt wird. Zentral für Zuboffs Analyse ist aber die weiträumige Ausforschung von Verhaltensweisen, die für eine Vielzahl von Zwecken ausge-

$22 S$. Zuboff (Fn. 1).

23 S. Zuboff (Fn. 1), 567.

24 Dies empfiehlt sich, wenn (wie in weiten Teilen der Wissenschaft üblich) zwischen Daten und Informationen unterschieden wird: Daten sind danach semantikfreie Träger von codierten Informationen. Bedeutung bzw. einen Sinn erhalten sie durch einen Vorgang der Übermittlung an einen Empfänger und wenn sie von diesem semantisch wahrgenommen werden.

25 S. Zuboff, (Fn. 1), 85 ff. 
wertet werden können, die sich von den ausgeforschten Personen oder Umständen, aus denen die Daten konkret erfasst wurden, lösen oder jedenfalls lösen können. Insofern scheint mir der Begriff „Ausforschungskapitalismus" treffender.

Die durch Ausforschung diverser Vorgänge wachsenden Bestände von proprietärem Verhaltensüberschuss werden nach der Analyse von Zuboff insbesondere zu Vorhersageprodukten verarbeitet, die in schnellem Tempo in für Verhaltensvorhersagen konzipierten Märkten gehandelt und in zahlreichen Geschäftsfeldern, aber auch in der Politik genutzt werden.

Eingangs ihres Buches umschreibt Zuboff die Form des neuen Kapitalismus - gewissermaßen seine Transformation - unter anderem wie folgt: ${ }^{26}$ „Erstens eine neue Marktform, die menschliche Erfahrung als kostenlosen Rohstoff für ihre versteckten kommerziellen Operationen der Extraktion, Vorhersage und des Verkaufs reklamiert; zweitens eine parasitäre ökonomische Logik, bei der die Produktion von Gütern und Dienstleistungen einer neuen globalen Architektur zur Verhaltensmodifikation untergeordnet ist; drittens eine aus der Art geschlagene Form des Kapitalismus, die sich durch eine Konzentration von Reichtum, Wissen und Macht auszeichnet, die in der Menschheitsgeschichte beispiellos ist; viertens Fundament und Rahmen einer Überwachungsökonomie ...; sechstens der Ursprung einer neuen instrumentären Macht, die Anspruch auf die Herrschaft über die Gesellschaft erhebt und die Marktdemokratie vor bestürzende Herausforderungen stellt ...; achtens eine Enteignung kritischer Menschenrechte, die am besten als Putsch von oben zu verstehen ist - als Sturz der Volkssouveränität."

Dies sind weitreichende Aussagen, die in ihrem sehr umfänglichen Buch näher ausgeführt werden. Dabei handelt es sich nicht um den Versuch einer allgemeinen Theorie des Kapitalismus, sondern um die übergreifende Analyse der vielfältigen Folgen der im Zuge der Digitalisierung eingesetzten neuen Geschäftsmodelle im IT-Bereich. Davon abgelöste Änderungen in weiteren Sektoren, etwa die Auswirkung der Digitalisierung in den verschiedenen industriellen Sektoren, sind nicht zentrales Thema ihrer Untersuchung.

Zuboffs Analyse konstatiert in ihrem Beobachtungsfeld einen Umsturz bestehender Marktmechanismen und als Folge deutliche Asymmetrien im Bereich von Wissen und Macht, gekoppelt mit erheblichen Auswirkungen auf die Funktionsweise rechtsstaatlicher und demokratischer Strukturen. Sie betont Einwirkungen auf Werte und Freiheiten und damit auch auf 
Möglichkeiten zur individuellen und kollektiven Gestaltung von Lebensverhältnissen. Ihre Analysen sind zugleich ein Beitrag zu der Bedeutung von Technik und Technikeinsatz als Mittel zur Herausbildung von Macht und können auch als Aufforderung verstanden werden, nach Gegenmitteln zu suchen - eine traditionelle Aufgabe des Rechts und der Rechtswissenschaft. ${ }^{27}$

Nur erwähnt sei, dass es auch andere kapitalismuskritische Analysen zum Bereich der Digitalisierung gibt, beispielsweise unter dem Schlagwort des „digitalen Kapitalismus“. ${ }^{28}$ Besonderheiten solcher Analysen bleiben hier ausgeklammert.

\section{Mustererkennung als Kernelement einer soziologischen Theorie der Gesellschaft}

Analysen zu digitalen Disruptionen und Transformationen erfolgen nicht notwendig primär markt- und machtbezogen. Anders angelegt ist die Analyse des Soziologen Armin Nassehi. ${ }^{29}$ Sein Erkenntnisinteresse zielt auf die Formulierung einer übergreifenden soziologischen Theorie der Gesellschaft. Er sieht in der Digitalisierung eine besonders ausgefeilte technische Lösung für ein Problem, das sich modernen Gesellschaften seit jeher stellt: Das Erkennen von bisher unsichtbaren Mustern, mit denen Akteure wie insbesondere Unternehmen, Staaten, Behörden, Verbände u.a. bei ihrem Handeln vorgehen. Die Digitalisierung biete Mustererkennungstechnologien mit transformationellem Potential, das es erlaube, menschliche Verhaltensweisen in bisher nicht möglicher Vielfalt und Masse zu erkennen, zu kontrollieren und zu regulieren. Big Data und KI machten die Komplexität der Gesellschaft in neuartiger Weise transparent. Die jetzt leichter erkennbaren gesellschaftlichen Regelmäßigkeiten, Strukturen und Muster seien das Material, aus dem digitale Techniken ihr ökonomisches, politisches und wissenschaftliches Potential schöpften.

Nassehi spricht hier anders als Zuboff nicht vom "Verhaltensüberschuss", sondern vom „Sinnüberschuss“, 30 den die Technik liefere, ohne dass dieser

27 Dazu s. - statt vieler - die Abschiedsvorlesung von A. Roßnagel zum Thema Technik, Recht und Macht. Aufgabe des Freiheitschutzes in Rechtsetzung und -anwendung im Technikrecht, MMR 2010, $222 \mathrm{ff}$.

28 So P. Staab, Digitaler Kapitalismus - Markt und Herrschaft in der Ökonomie der Unknappheit, 2019. S. auch N. Srnicek, How Will Capitalism End?, 2018.

29 A. Nassehi (Fn. 1).

30 A. Nassehi (Fn. 1), 264 ff. 
mit den Praktiken der Nutzer der Digitalisierung zu tun habe. Er lege Muster frei, die für unterschiedliche Anwendungen von Interesse sein könnten. Digitaltechnik sei eine Technik, die stets mehr bedeute, als sie vordergründig tue. In terminologischer Hinsicht möchte ich anmerken, dass der Begriff Sinnüberschuss nicht an Daten als solche - die als kodierte Informationen isoliert keinen Sinn vermitteln ${ }^{31}$ - anknüpft, sondern an ihre Nutzung mittels einer Technik, die durch Verarbeitung von Daten und ihrer Kombination mit anderen in spezifischen Kontexten Informationen weiterer Art - etwa über Verhaltensmuster - vermittelt. Eine der wesentlichen Änderungen gegenüber früher sei es, dass es den Bürgerinnen und Bürgern weitgehend nicht (mehr) möglich sei, sich einer Erhebung ihrer Daten zu verweigern und dass die Digitalisierung unendliche Möglichkeiten zur Kombination und Rekombination von Daten schaffe, darunter auch von Daten, die bei der Erhebung gar nicht zur Verknüpfung vorgesehen gewesen seien. ${ }^{32}$

Die damit verbundenen Veränderungen von Machtverhältnissen und die ergänzend zur Verfügbarkeit der Technologien bedeutsamen ökonomischen Steuerungsfaktoren und Auswirkungen auf die demokratische Ordnung sieht er, macht sie aber ebenso wie die mit der Digitalisierung verbundenen spezifischen Chancen, Risiken und sonstigen Disruptionspotentiale nicht eigenständig zum Thema. Er formuliert aber eine Grundlage zur Erfassung auch solcher Wirkungen und damit zugleich zu deren gesellschaftspolitischer Bewertung. Die Herausarbeitung der allgemeinen Bedeutung von Mustererkennungen für die Gesellschaft - wie auch für die Funktionsweise digitaler Techniken als solchen - und speziell für die durch die Digitalisierung bedingten Steigerungen der Möglichkeiten zur Erfassung von Mustern kann als Grundlage zur Analyse ihrer ökonomischen, politischen, kulturellen und wirtschaftlichen Nutzungen dienen, darunter auch der Einwirkungen auf Machtverhältnisse. Nassehis Einsichten können mittelbar auch Anregungen für Überlegungen zur Art der Erfüllung regulatorischer Aufgaben im Umgang mit den Folgen des Einsatzes der Mustererkennung geben.

31 S. auch o. unter Fn. 24.

32 Näher A. Nassebi (Fn. 1), 143 f, 259 f., 285 f. 


\section{Ein Sonderfall: Ausforschungs- und Überwachungstotalitarismus - Das Modell China}

Der Vollständigkeit halber möchte ich ergänzend auf einen grundlegend anderen Umgang mit der digitalen Transformation, als den etwa in Deutschland beobachtbaren, eingehen: den Einsatz digitaler Techniken in China zur Stabilisierung einer totalitären Regierungsform und zur Steuerung der Wirtschaft sowie zur Verhaltensbeeinflussung der dort lebenden Menschen. Die Nutzung der Digitalisierung ist darüber hinaus Teil der chinesischen Strategie, weltweit Einfluss zu gewinnen - der Bau der sog. zweiten Seidenstraße ist nur ein besonders sichtbares Zeichen infrastruktureller und wirtschaftlicher Expansion in vielen Teilen der Welt. ${ }^{33}$ Die Regierung versteht ihr Modell als eine Alternative zu dem der westlichen Demokratie und ist bemüht, es in andere Staaten - insbesondere solche mit totalitären Strukturen - zu exportieren.

Im Folgenden gehe ich nur auf einen Teilaspekt der chinesischen Strategie ein, nämlich den Einsatz digitaler Technologien zum Aufbau eines umfassenden Systems des Social Scoring der Bevölkerung. Für dieses System werden Daten der Verwaltung, der Gerichte, aber auch privater Unternehmen (etwa der Kommunikationsintermediäre, Webportale, Internetmehrwertdienste), Ergebnisse staatlicher Überwachung sowie Informationen durch Nachbarn und Mitarbeiter und ferner eingeforderte Selbsteinschätzungen der Betroffenen genutzt. Angestrebt wird eine umfassende Verbunddatei solcher Daten.

Das seit 2014 in mehreren Versuchsfeldern durchgeführte und ab Ende 2020 für ganz China vorgesehene Social Scoring System/Social Credit System $^{34}$ ist zum einen ökonomisch motiviert, nämlich durch das Ziel, das Wirtschaftssystem in China zu fördern und insbesondere die Markteffizienz zu erhöhen (und deshalb den Unternehmen zu ermöglichen, sich viele

33 Zur chinesischen Gesamtstrategie s. C. Hamilton/M. Oblberg, Die lautlose Eroberung. Wie China westliche Demokratien unterwandert und die Welt neu ordnet, 2020.

34 Hierzu Y. Chen/A. S. Y. Cheung, The Transparent Self Under Big Data Profiling: Privacy and Chinese Legislation on the Social Credit System, in: The Journal of Comparative Law 12, 2017, 356; R. Creemers, China's Social Credit System: An Evolving Practice of Control, 2018, https://ssrn.com/abstract=3175792; X. Dai, Toward a Reputation State: The Social Credit System Project of China, 2018, https:// ssrn.com/abstract=3193577. B. Kolany-Raiser/T. Radtke, Ich sammele, also bin ich (Social Credit) - das Szenario eines allumfassenden Bonitätssystems am Beispiel Chinas, in: T. Hoeren (Hrsg.), Phänomene des Big-Data-Zeitalters, 2019, 121 ff. S. auch $S$. Zuboff (Fn. 1), $451 \mathrm{ff}$. 
marktrelevante Daten verfügbar zu machen). Kommerziell orientierte Unternehmen - darunter vor allem, aber nicht nur marktbeherrschende ITUnternehmen wie die der Alibaba-Gruppe (sie verfügt u. a. über diverse Handelsplattformen und das weit verbreitete Online-Bezahlsystem Alipay) oder die der Tencent Holding (u. a. soziale Netzwerke, Nachrichtendienste, Online-Spiele) arbeiten zugleich eng mit staatlichen Institutionen und der kommunistischen Partei zusammen. Auch dafür erheben sie die Daten und verarbeiten/verknüpfen sie für diverse Auswertungen.

Denn es geht bei dem Social Scoring System insbesondere darum, staatliche und gesellschaftliche Stabilität zu sichern und dafür das Sozialverhalten der Menschen an bestimmten Wertvorgaben auszurichten (benannt werden etwa Ehrlichkeit, Verlässlichkeit, Integrität, Sauberkeit, Rechtstreue, Verantwortlichkeit in der Familie etc.) und staatliche und gesellschaftliche Stabilität zu sichern. Dem dient u. a. die Förderung von gesellschaftlicher Harmonie, die Unterdrückung möglicher Proteste und vor allem die Sicherung einer der kommunistischen Programmatik verpflichteten Wertebildung in der Bevölkerung. Zu diesem Zwecke wird die Beachtung der erwähnten sozialen Normen sowie politisches Wohl- und Fehlverhalten systematisch beobachtet und registriert. Auch gibt es unter Bezugnahme auf die erworbenen oder verfehlten Social Scoring Punkte eine Vielfalt von Möglichkeiten positiver oder negativer Sanktionen.

Nun ist gegenwärtig höchst unwahrscheinlich, dass ein ähnlich umfassendes Ausforschungs-, Überwachungs- und Sanktionssystem in funktionierenden rechtsstaatlichen Demokratien wie Deutschland eingerichtet wird oder absehbar geschaffen werden könnte. Technisch möglich wäre der Einsatz digitaler Technologien zu solchen Zwecken allerdings überall, sofern die dafür erforderlichen digitalen Infrastrukturen bestehen, so dass es zumindest nicht fern liegt, die Möglichkeiten digitaler Transformation zumindest illustrationshalber bis hin zu dem chinesischen Modell zu erfassen. Sowohl die rechtlichen Vorkehrungen als auch die kulturellen Traditionen und Werthaltungen der Bevölkerung dürften gegenwärtig allerdings in funktionierenden westlichen Demokratien stark genug sein, um sich diesem Modell zu verweigern. Allerdings: Wären solche Überwachungssysteme schon in der Zeit des Nationalsozialismus verfügbar gewesen, wären sie vermutlich auch in Deutschland genutzt worden. Angesichts von Ansätzen rechtsstaatlicher Erosionen gegenwärtig in der Türkei, Ungarn, Polen oder Rumänien scheint es auch für europäische Staaten wichtig zu sein, wachsam zu bleiben und im Zuge der Digitalisierung nicht totalitären Versuchungen zu erliegen, sondern für möglichst wirksame Gegenvorkehrungen zu sorgen. 


\section{Zwischenfazit}

Für den gegenwärtig begonnenen und noch keineswegs abgeschlossenen, durch Digitalisierung geprägten Wandlungsprozess von Wirtschaft, Staat und Gesellschaft drängen sich Parallelen zu den von Marx und Polanyi untersuchten Typen grundlegender Änderungen auf: Der Übergang vom Feudalismus einerseits und von der Agrarwirtschaft andererseits zu grundlegenden Veränderungen der jeweiligen Wirtschafts- und Gesellschaftsform. Der für die Gegenwart häufig gewählte Vergleich der Bedeutung der Industrialisierung mit der der Digitalisierung weist jedenfalls in eine solche Richtung.

Das chinesische Vorhaben einer auf digitaler Totalkontrolle aufbauenden Staats- und Gesellschaftsform liegt insofern auf einer anderen Ebene, als es nicht Produkt einer durch veränderte Rahmenbedingungen angestoBenen allmählichen Veränderung ist. Es handelt sich um ein politisch gezielt gesteuertes Vorhaben zum Erhalt und Weiterbau einer totalitären Staats- und Gesellschaftsform, das - wie erwähnt - auch als Gegenmodell zu rechtsstaatlichen Demokratien proklamiert wird. Die durch Digitalisierung neu erschlossenen Möglichkeiten der Überwachung und Steuerung sind ein zielorientiertes Instrument zum Ausbau des totalitären Modells.

Die oben (unter 2) erwähnte Analyse von Nassehi hat einen explizit theoretischen Anspruch. Sie zielt auf eine nicht auf bestimmte Phasen der historischen Entwicklung bezogene, übergreifende soziologische Theorie der Gesellschaft. Dafür hat das für digitale Techniken wichtige Element der Mustererkennung Anregungen gegeben: Damit werde ein Grundproblem moderner Gesellschaften und damit verbundener politischer Steuerung verdeutlicht: Das Erkennen von Handlungsmustern und deren Analyse auch als Grundlage für Einwirkungen auf wirtschaftliche, politische, kulturelle u. a. Entwicklungen.

Auf wieder anderen Ebenen liegen solche Wandlungsprozesse, die mit grundlegend veränderten Sichtweisen verbunden sind. Hierzu zähle ich die besonders weitreichende, mit dem Namen Kopernikus verbundene Neuausrichtung des mittelalterlichen Denkens über die Welt. Nicht mit einer ähnlichen Reichweite, aber doch mit einem strukturell vergleichbaren Ansatz versehen sind die mit den Namen Kubn und Fleck verbundenen Einsichten über die Bedeutung von Paradigmen und Denkstilen (auch) für Wandlungsprozesse. In die Reihe transformativer Veränderungen im Denken und Handeln ordne ich auch die wachsende Bedeutung konstruktivistischer Perspektiven auf Wirklichkeit und damit zugleich das Bemühen um ein modernes Verständnis von Rationalität. Analysen, Vorhersagen, 
Entscheidungen $\mathrm{u}$ ä. unter Nutzung digitaler Techniken sind Prototypen konstruktivistischen Vorgehens.

Solche oder andere Versuche zur Einordnung der gegenwärtigen Entwicklung und ihres Verständnisses in größere Zusammenhänge mögen Anregungen für weitere Hypothesen oder für Widerspruch geben, sollen hier aber nicht weiterverfolgt werden.

\section{Wirkungsdimensionen transformativer Wandlungen}

Erneut sei auf die Annahme verwiesen, dass die digitale Transformation in weite Teile von Wirtschaft, Kultur, Politik, öffentlicher und privater Kommunikation hineinwirkt und erhebliche Auswirkungen auf die Gesellschaft und Wirtschaft der Gegenwart hat. ${ }^{35}$ Das Erkennen von verschiedenen Ebenen von Wirkungen und Vorgehensweisen beim Umgang mit ihnen ist wesentlich auch für die Reaktion des Rechts auf die digitale Transformation.

In der wissenschaftlichen Literatur, insbesondere der ökonomisch orientierten, hat sich weithin zur allgemein einsetzbaren Klassifizierung unterschiedlicher Wirkungsdimensionen von Entscheidungen und Verhalten das Begriffstrio Output, Outcome und Impact durchgesetzt. ${ }^{36}$ Dieses Trio lässt sich auch zur Erfassung der mit der digitalen Transformation verbundenen Wirkungen nutzen.

$\mathrm{Zu}$ den Wirkungen gehören selbstverständlich die durch den Einsatz neuer Technologien in je unterschiedlichen Bereichen ermöglichten Verfahren, Produkte, Einsichten und Dienste - also der konkrete Output. Hinzu treten die Wirkungen bei unmittelbar oder mittelbar Betroffenen, etwa bei den Nutzern von digitalen Diensten: Der Impact als Mikrowirkung. Drittens ist der Outcome als darüber hinausreichende, auch längerfristige Veränderung in den betroffenen gesellschaftlichen Bereichen wichtig, also die Wirkung auf der Makroebene. Dazu gehören auch die Auswirkungen auf Marktstrukturen, auf die demokratische Ordnung, auf die Leistungsfähigkeit rechtsstaatlicher Schutzvorkehrungen, auf die Gewinnung von weiter verwertbarem Wissen, auf die Entwicklung des kulturellen Bewusstseins, auf die öffentliche Kommunikation u. a. m.

35 S. dazu etwa A. Reckwitz, Das Ende der Illusionen. Politik, Ökonomie und Kultur in der Spätmoderne, 2020, insbes. $133 \mathrm{ff}$., $162 \mathrm{ff}$.

$36 \mathrm{Zu}$ diesen Begriffen s. F. Nullmeier, Input, Output, Outcome, Effektivität und Effizienz in: B. Blanke/F. Nullmeier/C. Reichard/G. Wewer (Hrsg.), Handbuch zur Verwaltungsreform, 4. Aufl., 2010, 357, $360 \mathrm{ff}$. 
Wirkungen in allen drei Dimensionen lassen sich in vielen bereichsspezifischen Anwendungsfeldern des Einsatzes digitaler Technologien feststellen. Ich nenne als Kandidaten beispielhaft die medizinische Forschung, Diagnose und Therapie, das Geschehen auf Finanzmärkten, Veränderungen im Arbeitsleben, im Handel, in der Mobilität sowie Möglichkeiten hoheitlicher Überwachung.

Als ein weiteres wichtiges Feld erwähne ich den Energiebereich, der zugleich ein Beispiel für die mögliche Ambivalenz der Wirkungen ist. Digitale Technologien haben die Entwicklung neuer Verfahren der Energiegewinnung, -speicherung, -verteilung und von Maßnahmen zur Steigerung der Effizienz des Energieverbrauchs gefördert. Mit den neuen Möglichkeiten sind aber auch neue Probleme verbunden. So darf nicht übersehen werden, dass die Informationstechnologien zum Teil hoch energieintensiv arbeiten - so etwa der Betrieb von Clouds, der Einsatz der BlockchainTechnologie oder das Streaming. Das alles ist mit dem Risiko verbunden, dass die durch die gegenwärtige Klimakrise angestoßenen Ansätze für neue Energieformen, zur Energieeinsparung bzw. zu vermehrter Effizienz der Energienutzung durch den Einsatz von Informationstechnologien in ihren Wirkungen zumindest teilweise konterkariert werden.

Für die Wirkungen der Digitalisierung sind auch Entgrenzungen, Konvergenzen und Vernetzungen verschiedener Art typisch. ${ }^{37}$ Dazu zählen die Trans- und Internationalität des Einsatzes digitaler Techniken. Ferner: im IT-Bereich verschwimmen die Grenzen zwischen Hardware, Software und Orgware, zwischen Anbietern und Nachfragern sowie zwischen Dienstleistungen unter Nutzung von IT-Infrastrukturen. Private und öffentliche Kommunikation werden verstärkt miteinander verwoben. Herkömmliche Vorstellungen über Privatheit und Öffentlichkeit erodieren, die Notwendigkeit von spezifischem Privatheitsschutz wird sogar zum Teil - so durch Anhängerinnen und Anhänger der sog. Post-privacy-Bewegung - bezweifelt. ${ }^{38}$

37 Dazu näher s. W. Hoffmann-Riem, Rechtliche Rahmenbedingungen für und regulative Herausforderungen durch Big Data, in: Ders. (Hrsg.), Big Data - Regulative Herausforderungen, 2018, 11, $36 \mathrm{ff}$.

38 Dazu s. statt vieler C. Heller, Post-privacy: Prima leben ohne Privatsphäre, 2011. Kritisch zu einer solchen Position P. Schaar, Das Ende der Privatsphäre, 2007. Differenzierend M. Klar, Privatsphäre und Datenschutz in Zeiten technischen und legislativen Umbruchs, in: Die öffentliche Verwaltung (DÖV) 2013, 103; V. BoehmeNeßler, Zwei Welten? Big Data und Datenschutz. Entwicklungslinien des Datenschutzes in der digitalen Gesellschaft, in: Archiv für Urheber- und Medienrecht 2015, 19, 24-27. Zur Problematik siehe auch die Beiträge in H. Hill/U. Schliesky (Hrsg.), Die Neubestimmung der Privatheit, 2014. 
In den Bereich von Entgrenzungen und Konvergenzen gehört auch dies sei besonders hervorgehoben - der Befund wachsender Ubiquität (Allgegenwärtigkeit) informationstechnischer Geräte, Infrastrukturen und nutzbarer Dienste (sog. Ubiquitous Computing): Datenerhebung,- verarbeitung und -nutzung sind zu einem integralen Bestandteil des Alltags geworden. ${ }^{39}$ Für die Verwobenheit von Interaktionen Online und Offline steht gegenwärtig - in kritischer Absicht - noch ein anderer Begriff: Onlife. ${ }^{40}$ Wenn unser Leben vielfach weder On- noch Offline ist, beginnt (so die These) die Bildung einer neuen Art von Welt - die Onlife-Welt. In ihr können Computersysteme die Menschen insbesondere von Entscheidungsnotwendigkeiten weitgehend freistellen, also menschliche Entscheidungen vorprägen ${ }^{41}$ oder gar ersetzen. Solche Entscheidungsentlastungen können als große Chance auf einen Gewinn an Lebensqualität beurteilt, aber auch kritisiert werden, insbesondere soweit die Betroffenen keine oder nur eine begrenzte Gelegenheit zur willentlichen Intervention haben. Mireille Hildebrandt spricht in diesem Zusammenhang von „Pre-emptive Computing Systems ${ }^{\text {“ }}{ }^{42}$ Das mit dem Einsatz solcher Technologien verbundene Unterlaufen bewusster Reflexion bewirke - so Hildebrandt -, dass der Mensch in

39 Statt vieler: L. Lipp, Interaktion zwischen Mensch und Computer im Ubiquitous Computing. Lit Verlag, 2004; M. Friedewald, O. Raabe, P. Georgieff u. a., Ubiquitäres Computing. Das „Internet der Dinge“ - Grundlagen, Anwendungen, Folgen, 2010; E. Peuker (Fn. 3) (Manuskript S. 25 f).

40 Dazu s. L. Floridi, Die 4. Revolution, 2015, $87 \mathrm{ff} ., 129 \mathrm{ff}$.; M. Hildebrandt, Smart Technologies and the End(s) of Law. Novel Entanglements of Law and Technology, 2016, $41 \mathrm{ff} ., 77 \mathrm{ff}$. Hildebrandt definiert die Onlife World als: „The hybrid life world composed of and constituted by combination of software and hardware that determine information flows and the capability to perceive and cognise one's environment which is run by means of an information and communication infrastructure (ICI) capable of pre-emptive computing, based on its tapping into the digital unconscious of big data space." Den von ihr vielfach genutzten Begriff "Digital Unconscious" definiert sie wie folgt: "The largely invisible big data space on which the onlife world and its ICI of pre-emptive computing depend, where inferences are thrown and applied, largely beyond the ambit of conscious reflexion." (261), s. a. $65 \mathrm{ff}$.

41 Dies erfasst auch die Vorprägung des Nutzerverhaltens in Social Media, das algorithmisch unbemerkt gesteuert wird, etwa durch personenbezogene, an früherem Nutzerverhalten ausgerichtete Filterung des Informationszugangs.

42 Zur Definition s. M. Hildebrandt (Fn. 40), 263. Gemeint sind insbesondere computerisierte Systeme, die Verhalten, insbesondere in Gestalt von Verhaltensmustern, erfassen, darauf aufbauend Verhaltensmöglichkeiten im Vorwege einschätzen und gleichzeitig Anstöße dafür zu geben, dass die Erwartung entsprechenden Verhaltens auch eingelöst wird. 
der Onlife-Welt vielfach und vermehrt zum „Digital Unconscious“, 43 zum Objekt unbewusster Steuerung, werde. Damit drohe ein Grundprinzip moderner Gesellschaften, die Autonomie im Handeln, (weiter) zu erodieren.

\section{Zur begrifflichen Systematisierung unterschiedlicher digital beeinflusster Entscheidungen}

Die eher subtile Beeinflussung von Entscheidungen im zuletzt beschriebenen Sinne betrifft einen Typ von Verhaltenssteuerung, bei dem die Entscheidungen durch die Ergebnisse des Einsatzes algorithmischer Systeme in einer Weise vorgeprägt sind, dass die Eigenbestimmung bei der Entscheidung zwar nicht ausgeschlossen, aber faktisch eingeschränkt ist: Die Auswahl zwischen verschiedenen Handlungsoptionen ist zwar möglich, der tatsächliche Entscheidungsspielraum und damit die Eigenbestimmung zur Entscheidung aber sind durch die subtile Einflussnahme faktisch limitiert. Die Datenethikkommission der Bundesregierung hat bei ihrem Versuch, unterschiedliche Entscheidungssituationen begrifflich zu kennzeichnen, ${ }^{44}$ hierfür den Begriff der algorithmengetriebenen Entscheidungen vorgeschlagen.

Die Kommission hat auch weitere hilfreiche Begriffe genutzt, um die unterschiedliche Einsetzbarkeit von Algorithmen für Entscheidungen darunter können auch rechtliche Entscheidungen fallen - zu verdeutlichen. Werden menschliche Entscheidungen nicht durch Algorithmen ersetzt, stützen die Entscheidungen sich aber auf algorithmisch berechnete (Teil-)Informationen - dies ist auch in der Praxis des Umgangs mit Recht durch Rechtsanwälte, Behörden und Gerichte schon jetzt häufig der Fall -, wird der Begriff der algorithmenbasierten Entscheidungen empfohlen. Soweit demgegenüber ein menschliches Handeln ausscheidet und stattdessen vollständig automatisiert vorgegangen wird, ${ }^{45}$ wird der Begriff der algorithmen-

43 Zu dieser Definition s. M. Hildebrandt (Fn. 40), 261, 263. S. ferner die Beispiele auf den Seiten 65 ff. (wie z.B. Enhanced Targeting, Attention Management).

44 Gutachten der Datenethikkommission, 2019, 24, 181.

45 Zur Möglichkeit automatisierter Entscheidungen s. etwa Art. 22 EU-DSGVO; $\$ 35$ a VerwVerfG. Speziell zu automatisierten Verwaltungsakten s. A. Berger, Der automatisierte Verwaltungsakt, NVwZ 2018, 1269 ff.; A. Guckelberger, E-Government: Ein Paradigmenwechsel in Verwaltung und Verwaltungsrecht?, in: VVDStRL 78, 2019, 235, 263 ff.; H. Kube, E-Government: Ein Paradigmenwechsel in Verwaltung und Verwaltungsrecht?, in: VVDStRL 78, 2019, 299, 301 ff., 307 ff.; 
determinierten Entscheidungen maßgebend. Alle drei Dimensionen können beispielsweise beim Einsatz von Legal Technology (s. u. X) auftreten.

Die begrifflichen Zuordnungen sind auch Anschauungsmaterial für den Befund der Veränderung der für den Umgang mit Recht maßgebenden Paradigmen bzw. Denkstile (zu letzteren s. o. I.5). Die teilweise oder vollständige Ersetzung der menschlichen Entscheidung oder die durch digitale Techniken bewirkte Einschränkung der Möglichkeit selbstbestimmter Entscheidung verändert die Konstruktion rechtlicher Wirklichkeit, beeinflusst das zugrunde gelegte Rationalitätskonzept und hat Rückwirkungen auf die Möglichkeit zur Sicherung der Legitimation der Entscheidung. Darauf wird zurück zu kommen sein.

\section{Transformation des Innovationsermöglichungsrechts}

Disruptive, transformativ wirkende Technologien sind ein wichtiges Thema und Mittel der Innovationsforschung. ${ }^{46}$ Dazu verweise ich auf das Referat von Ingo Schulz-Schaeffer. ${ }^{47}$ Digital fundierte Innovationen sind selbstverständlich auch Gegenstand der rechtswissenschaftlichen Innovationsforschung. ${ }^{48}$ Moderne Gesellschaften benötigen Räume für Innovationen, also Innovationsoffenheit, aber auch Aufmerksamkeit nicht nur für die möglichen Chancen für Verbesserungen, sondern auch für Risiken und für ggf. innovative Gegenmittel zu deren Vermeidung oder Minimierung. Gefordert ist daher auch eine in dem gesellschaftlichen Umfeld, in sozialen Normen und in der Rechtsordnung verankerte Innovationsverantwortung der Akteure. ${ }^{49}$ Auch bedarf es der Herausbildung von Strukturen, die Raum für die Realisierung von Innovationen durch potentiell Akteure jeglicher Art schaffen.

M. Stegmüller, Vollautomatische Verwaltungsakte - eine kritische Sicht auf die neuen $\$ 24$ I 3 und $\$ 35$ a VwVfG, NVwZ 2018, $353 \mathrm{ff}$.

46 Allgemein dazu die Beiträge in B. Blättel-Mink/I. Schulz-Schaeffer/A. Windeler (Hrsg.), Handbuch der Innovationsforschung. Sozialwissenschaftliche Perspektiven, 2020.

47 I. d. B.

$48 \mathrm{Zu}$ dieser Richtung allgemein s. m. w. Hinw. W. Hoffmann-Riem, Innovation und Recht - Recht und Innovation. Recht im Ensemble seiner Kontexte, 2016; und die Beiträge in: Ders. (Hrsg.), Innovationen im Recht, 2016.

$49 \mathrm{Zu}$ den Begriffen s. W. Hoffmann-Riem, Innovationsoffenheit und Innovationsverantwortung durch Recht. Aufgaben rechtswissenschaftlicher Innovationsforschung, AöR 131, 2006, 255 ff. 
Innovationsoffenheit und Innovationsverantwortung sind wichtige Pole auch der transformativen Elemente des IT-bezogenen Rechts, das die Wahrnehmung der Chancen der Digitalisierung ermöglichen, aber Risiken minimieren oder gar ausschließen soll, kurz: für ein als Innovationsermöglichungsrecht konzipiertes Recht. ${ }^{50}$ Dafür ist es wichtig, reicht es aber nicht, rechtlich Freiräume für Ideen, für das Ausprobieren von Neuem und seiner Anwendung und für die ökonomische Verwertung zu schaffen. Es sind auch die positiv oder negativ zu bewertenden Folgen in den Wirkungsebenen Output, Impact und Outcome zu berücksichtigen und es ist darauf hinzuwirken, dass die Verwirklichung von Chancen auch real ermöglicht und Risiken beherrschbar gehalten werden. So müssen Fehlentwicklungen korrigierbar sein und bei Bedarf auch korrigiert werden.

Für Innovationsoffenheit und Innovationsverantwortung im Kontext der Digitalisierung zu sorgen, ist eine Aufgabe auch der zur Sicherung der Wirksamkeit der Rechtsordnung berufenen Akteure (Politiker, Unternehmen, Verwaltungsbeamte, Gerichte, Verbände, ferner Vertreterinnen und Vertreter der Zivilgesellschaft sowie die einzelnen Bürgerinnen und Bürger). Es bedarf nicht nur eines auf die Besonderheiten der Digitalisierung abgestimmten rechtlichen Instrumentariums, sondern auch eines darauf eingestellten gesellschaftlichen Umfelds und dafür geeigneter wirtschaftlicher Strukturen und kultureller Orientierungen.

Kurz: Entscheidend ist die Art der auf die digitale Transformation abgestimmten Governance. Insofern geht es um die Fortentwicklung der (bisher allerdings nur begrenzt befriedigenden) Bemühungen um eine anspruchsvolle Internet-Governance. ${ }^{51}$ Sie müsste heute zur IT-Governance ausgeweitet werden, nämlich unter Einbeziehung der Governancestruktu-

50 Dazu s. W. Hoffmann-Riem (Fn. 48), S. 33 ff.

51 Dazu s. etwa $D$. Drissel, Internet-Governance in a Multipolar World: Challenging American Hegemony, 19 Cambridge Review of International Affairs, 2006, 105 et seq.; Kurbalija, An Introduction to Internet-Governance, 5. Aufl., 2012; R. H. Weber, Realizing a New Global Cyberspace Framework: Foundations and Guiding Principles, 2014; Ders. Kommentierung von Internet-Governance, in R.H. Weber/T. Hoeren/B. Holznagel (Hrsg.), Multimedia-Recht, Stand 51. El. 2020. Zur Definition von Internet-Governance s. Report of the Working Group on Internet-Governance, June 2005, http://www.wgig.org/docs/WGIGREPORT.pdf (Stand: 2014): „Internet-Governance“ als die „Entwicklung und Anwendung durch Regierungen, den Privatsektor, die akademische/technische Gemeinschaft und die Zivilgesellschaft, in ihren jeweiligen Rollen, von gemeinsamen Prinzipien, Normen, Regeln, Vorgehensweisen zur Entscheidungsfindung und Programmen, welche die Weiterentwicklung und die Nutzung des Internet beeinflussen“. 
ren im Hinblick auf die Prozesse der Digitalisierung auch außerhalb des Internet.

\section{Zur Schutz-und Gewährleistungsfunktion von Recht}

Aufträge zur rechtlichen Ausgestaltung des transformativen Innovationsgeschehens sind mit der seit langem anerkannten Schutz- und Gewährleistungsfunktion von Recht verbunden ${ }^{52}$ - vermittelt einerseits über abwehrrechtliche, insbesondere gegen den Staat gerichtete subjektive Rechte der Bürgerinnen und Bürger, ferner durch die über die objektivrechtlichen Gehalte vieler Grundrechte ${ }^{53}$ vermittelten Ausgestaltungs- und Schutzaufträge. ${ }^{54}$ Solche auf die Gewährleistung von Freiheitsrechten, und zwar auch im Verhältnis Privater untereinander, gerichteten objektiv-rechtlichen Gehalte von Grundrechten finden sich im Übrigen nicht nur in nationalen, etwa den deutschen, Grundrechtsnormen, sondern werden begrenzt, aber zunehmend ebenfalls im Bereich der EU-Grundrechtecharta sowie der Europäischen Menschenrechtskonvention und in einzelnen völkerrechtlichen Abkommen anerkannt. ${ }^{55}$ Aufträge zum Schutz kollektiver

52 Beispiele dafür gibt es viele. Ich greife eine thematisch einschlägige Publikation heraus, bezogen auf die individuellen Möglichkeiten internetbasierter Kommunikation: M. Oermann (Fn. 4).

53 Grundlegend für diese Funktion von Grundrechtsnormen ist die Entscheidung des Bundesverfassungsgerichts BVerfGE 7, 198, $203 \mathrm{ff}$. S. ferner BVerfGE 42, 143,148; 89, 214, 229; 103, 89, 100; 137, 273, 313, Rn. 109; 148, 267, Rn. 32.

54 Allgemein zu objektiv-rechtlichen Grundrechtsgehalten und Schutzpflichten s. M. Dolderer, Objektive Grundrechtsgehalte, 2000; L. Jaeckel, Schutzpflichten im deutschen und europäischen Recht, 2010; P. Szeczekalla, Die sogenannten grundrechtlichen Schutzpflichten im deutschen und europäischen Recht, 2002. S. auch Fn. 55.

55 S. dazu T. Maraubn, Sicherung grund- und menschenrechtlicher Standards gegenüber neuen Gefährdungen durch private und ausländische Akteure, in: VVDStRL, Bd. 74, 2015, 373 ff.; C. Teetzmann, Schutz vor Wissen? Forschung mit doppeltem Verwendungszweck zwischen Schutzpflichten und Wissenschaftsfreiheit, 2020, 148 ff., 369 ff. (speziell zur Biotechnologie). Mit Bezug speziell zur Internetkommunikation s. A. Fischer-Lescano, Der Kampf um die Internetverfassung: Rechtsfragen des Schutzes globaler Kommunikationsstrukturen von Überwachungsmaßnahmen, JZ 2014, 965 ff.; U. Schliesky/C. Hoffmann/A. D. Luch/S. E. Schulz/K. C. Borchers, Schutzpflichten und Drittwirkungen im Internet: Das Grundgesetz im digitalen Zeitalter, 2014; N. Marsch, Das europäische Datenschutzgrundrecht. Grundlagen - Dimensionen - Verflechtungen, Tübingen, 2018; S. V. Knebel, Die Drittwirkung der Grundrechte und -freiheiten gegenüber Privaten. Regulierungsmöglichkeiten sozialer Netzwerke, 2018. 
Rechtsgüter werden inhaltlich auch durch die Staatszielbestimmungen wie die der Rechtstaatlichkeit, Demokratie und Sozialstaatlichkeit angeleitet.

An der aktuellen verfassungsgerichtlichen Rechtsprechung fällt auf, dass das BVerfG erwägt, die seit langem anerkannte objektivrechtliche Dimension von Grundrechten und die damit gerechtfertigte mittelbare Drittwirkung von Grundrechten im Verhältnis Privater untereinander in Richtung auf die Möglichkeit unmittelbarer Wirkungen der Grundrechte jedenfalls in spezifischen Konstellationen auszudehnen. ${ }^{56}$ Für die Begründung solcher Weiterungen sei mitentscheidend, ob die Grundrechtsbindung Privater einer Grundrechtsbindung des Staates nahe oder gleich komme. Im Bereich der Kommunikation kämen Gewährleistungsaufträge insbesondere in Betracht, wenn private Unternehmen die Bereitstellung schon der Rahmenbedingungen öffentlicher Kommunikation selbst übernehmen und damit in Funktionen eintreten, die früher - wie die Sicherstellung der Post- und Telekommunikationsdienstleistungen - dem Staat als Aufgabe der Daseinsvorsorge zugewiesen waren. ${ }^{57}$

Diese Überlegungen sind von der 2. Kammer des Ersten Senats i. J. 2019 ausdrücklich auch auf Betreiber sozialer Netzwerke im Internet bezogen worden. ${ }^{58} \mathrm{Da}$ diese Entscheidung in einem Eilverfahren erging, bestand für das Gericht allerdings kein Anlass, diese Frage definitiv zu klären. Es fällt aber auf, dass das Gericht - obwohl es für die Entscheidung darauf nicht ankam (Juristen sprechen hier von einem obiter dictum) - ausdrücklich ausführte: „Ob und gegebenenfalls welche rechtlichen Forderungen sich insoweit auch für Betreiber sozialer Netzwerke im Internet" (konkret ging es um Facebook) „etwa in Abhängigkeit vom Grad deren marktbeherrschender Stellung, der Ausrichtung der Plattform, des Grads der Angewiesenheit auf eben jene Plattform und den betroffenen Interessen der Plattformbetreiber und sonstiger Dritter - ergeben, " sei jedoch in der Rechtsprechung des BVerfG noch nicht abschließend geklärt. Noch deutlicher wird das Gericht wenig später in der Entscheidung des Ersten Senats zum „Recht auf Vergessen I“. Es heißt dort - wiederum als obiter dictum -: Bei der Bestimmung der Reichweite der mittelbaren Grund-

56 Zur Diskussion der im sogleich folgenden Text erwähnten sowie weiterer Entscheidungen s. B. Raue, Meinungsfreiheit in sozialen Netzwerken, JZ 2018, 94; F. Michl, Situativ staatsgleiche Grundrechtsbindung privater Akteure, JZ 2018, 910; A. Hellgardt, Wer hat Angst vor der unmittelbaren Drittwirkung?, JZ 2018, $901 \mathrm{ff}$;; S. Muckel, Wandel des Verhältnisses von Staat und Gesellschaft - Folgen für Grundrechtstheorie und -dogmatik, VVDStRL79, 2020, 245, 276.

57 BVerfGE 128, 226, $249 \mathrm{f}$.

58 BVerfG NJW 2019, 1935, Rn 15 unter Verweis auf BVerfGE 148, 267, 283 f. 
rechtswirkung „können insbesondere auch die Unausweichlichkeit von Situationen, das Ungleichgewicht zwischen sich gegenüber stehenden Parteien, die gesellschaftliche Bedeutung bestimmter Leistungen oder die soziale Mächtigkeit einer Seite eine maßgebliche Rolle spielen." ${ }^{99}$ Solche Ausführungen können als Andeutung verstanden werden, dass die durch die Digitalisierung bedingte Asymmetrie in den Machtverhältnissen - wie sie beispielsweise Shoshana Zuboff thematisiert - transformative Folgen in der Reichweite des Grundrechtsschutzes haben könnte. Verbindlich entschieden ist dies allerdings noch nicht.

Wenn zur Beurteilung der Reichweite des Grundrechtsschutzes hier und möglicherweise auch in zukünftigen Fällen die Macht der Akteure maßgebend werden sollte, würde dies auch andere Bereiche als die des Kommunikationssektors erfassen können. So formuliert Alexander Roßnagel: „Heute ist der Staat noch immer eine mächtige Instanz, aber bei weitem nicht mehr die einzige und in manchen Bereichen auch nicht mehr die stärkste. Heute machen große Konzerne durch ihren Technikeinsatz im Bereich der Energie, Chemie, Pharmazie und vor allem der Information und Kommunikation dem Staat seine Rolle als Träger von Macht streitig. Im Internet übernehmen sie sogar staatliche Funktionen wie das Setzen und Vollziehen von Regeln des Zusammenlebens. Sie entscheiden über die Verwirklichungsbedingungen von Grundrechten und damit über die Grundlagen individueller und gesellschaftlicher Freiheit. Den gesellschaftlichen Informations- und Kommunikationsraum beherrschen sie sogar stärker als bisher der Staat den analogen. “60

\section{Private Selbstgestaltung und Selbstregulierung und die Notwendigkeit transformativer regulativer Umbegungen}

\section{Privatisierung und Deregulierung der Telekommunikation}

Die Sicherung von Individual- und Gemeinwohl ist eine gemeinsame Aufgabe von Staat und Gesellschaft. Infolge der anhand neoliberaler Konzepte seit mehreren Jahrzehnten betriebenen, insbesondere von der EU forcierten, Privatisierung ehemals staatlicher Aufgaben und der damit einhergehenden Deregulierung vieler Bereiche ist das Tätigkeitsfeld staatlicher Or-

59 BVerfG NJW 2020, 300, Rn 77.

60 A. Roßnagel, Technik, Recht und Macht, MMR 2020, 222. 
gane zurückgedrängt worden. ${ }^{61}$ Das betrifft, wie das BVerfG ausdrücklich erwähnt, ${ }^{62}$ den früher in vielen Staaten in staatlicher Verantwortung gelegenen klassischen Telekommunikationsbereich. Ungeachtet der großflächigen digitalen Transformation und der mit ihr verbundenen neuen Betätigungs- und Geschäftsfelder und des damit verkoppelten Ausbaus privater Macht ist diese Privatisierung bisher nicht wieder rückgängig gemacht worden. Die primäre Steuerung der Entwicklung des IT-Bereichs und in der Folge die Hauptverantwortung für die Nutzung seiner Chancen sowie für die Bewältigung der mit der Digitalisierung verbundenen Probleme und insbesondere für den Umgang mit Risiken liegen daher nicht oder nur sehr begrenzt beim Staat, sondern vorrangig bei den im IT-Bereich tätigen Unternehmen und - in Grenzen - bei den Nutzern ihrer Produkte und sonstigen Leistungen.

\section{Exkurs: Kommerzialisierung insbesondere des Internet}

Die Möglichkeit privater Selbstgestaltung und -regelung war bei der Entwicklung des Internet - und damit auch beim Startschuss für die rasante Fortentwicklung der digitalen Transformation - vor allem in den USA erheblich stärker ausgeprägt als in Deutschland und anderen europäischen Staaten. Das Internet wurde - ungeachtet seiner Förderung durch das amerikanische Militär und amerikanische Bildungsinstitutionen - von vielen als privat gestalteter Kommunikationsort der Freiheit, der Emanzipation und selbstregulativen Entfaltung, ja der Möglichkeit auch einer anarchischen Entwicklung verstanden. ${ }^{63}$ Dieser Einschätzung stand die zunehmende Kommerzialisierung des Internet angesichts der vorherrschenden neoliberalen Marktkonzeption jedenfalls zunächst nicht grundsätzlich entgegen. Die weitere Entwicklung allerdings untergrub dieses Ideal schnell. Das Ergebnis war eine "privatwirtschaftliche Landnahme“ des Internet, ${ }^{64}$

61 Allgemein zur Privatisierung und Deregulierung A. Voßkuble, in: W. HoffmannRiem/E. Schmidt-Aßmann/A. Voßkuhle (Hrsg.), Grundlagen des Verwaltungsrechts, Bd. 1, 2. Aufl. 2012, 1, 46 ff. m. w. Hinw.

62 BVerfGE 128, 226, $249 \mathrm{f}$.

63 Besonders prononciert dazu J. P. Barlow, A declaration of the independence of cyberspace. Electronic Frontier Foundation, 1996, https://www.eff.org/cyberspaceindependence.

64 So U. Dolata, Plattform-Regulierung. Koordination von Märkten und Kuratierung von Sozialität im Internet, Berliner Journal für Soziologie, 2020, https://doi. org/10.1007/s11609-020-00403-9, unter 1. 
die zu massiven Kommerzialisierungsprozessen und in der Folge wachsender Asymmetrie von Marktmacht und Einflussmacht führte.

Ulrich Dolata fasst die Entwicklung wie folgt zusammen. Die „Konzerne entwickeln und betreiben mit ihren Plattformen die wesentlichen technischen Infrastrukturen und Dienste des Webs, auf die nicht nur private Nutzer, sondern auch viele Unternehmen und öffentliche Einrichtungen häufig zugreifen (müssen). Als quasi-hoheitliche Akteure kontrollieren sie die zentralen Zugänge zum Internet, strukturieren und observieren die Bewegungsmöglichkeiten der Nutzer, kuratieren und korrigieren in großem Stil Inhalte, Informationsflüsse und Diskussionen. Als strukturbildende Wirtschaftsakteure arbeiten sie an der möglichst lückenlosen Erfassung, Verarbeitung und Inwertsetzung der Datenspuren, die die Nutzer im Web hinterlassen und haben damit einen großflächigen Prozess der kommerziellen Vermessung und Kommodifizierung sozialer Aktivitäten und Beziehungen im Online-Bereich in Gang gesetzt, der in der Welt vor dem Internet undenkbar gewesen wäre. Darüber hinaus agieren sie nicht mehr bloß als führende und akzentsetzende Marktteilnehmer, sondern unterhalten und regulieren auch eigene Märkte und Arbeitsbeziehungen, deren Teilnehmerkreis zum Teil weit über ihren Unternehmenszusammenhang hinausreicht". ${ }^{65}$

\section{Zur Art der Selbstgestaltung und Selbstregulierung}

Prägend für die geschaffenen Strukturen und das Handeln der Unternehmen ist eine weitgehende Selbstgestaltung der Geschäftsmodelle, der technologischen und soziotechnischen Infrastrukturen, der Beziehungen zu anderen Unternehmen und zu den diversen Nutzern. Als machtvolle Akteure können die Unternehmen eigenständig auch Regeln schaffen (Selbstregulierung $)^{66}$ oder sich zusammen mit anderen Akteuren kollektiv über Regeln verständigen, und zwar ohne dass dies demokratischer Kontrolle unterliegt. In der Folge haben sie schon jetzt vielfach eigene normative Ordnungen geschaffen. ${ }^{67}$ Diese befinden sich nicht nur in den von den

65 U. Dolata, (Fn. 64) unter 1, m. Hinw. auf weitere eigene Veröffentlichungen.

$66 \mathrm{Zu}$ diesem und weiteren Begriffen s. W. Hoffmann-Riem, Selbstregelung, Selbstregulierung und regulierte Selbstregulierung im digitalen Kontext, in: $M$. Fehling/U. Schliesky (Hrsg.), Neue Macht- und Verantwortungsstrukturen in der digitalen Welt, 2018, $27 \mathrm{ff}$.

67 D. Wielsch, Die Ordnung der Netzwerke. AGB - Code - Community Standards, in: M. Eifert/T. Gostomzyk (Hrsg.) Netzwerkrecht. Zur Zukunft des NetzDG und 
Unternehmen formulierten Allgemeinen Geschäftsbedingungen, sondern sie haben auch die Gestalt von Gemeinschaftsstandards oder von Verhaltensregeln.

Solche Regeln können mittelbar auch Wirkungen für Dritte entfalten. Ein Beispiel ist das internationale Robot-Exclusion-Standardprotokoll (REP). ${ }^{68}$ Es betrifft die Möglichkeit der Betreiber von Websites, diese oder Teile davon gegenüber denjenigen Web-Crawlern (Robots) zu sperren, welche die Standards des REP befolgen. So werden die Suchmaschinen, welche diese Web-Crawler für ihre Indexierung nutzen, daran gehindert, diese Inhalte zugänglich zu machen. Dem zunächst durch ein Expertengremium erarbeiteten REP haben sich verschiedene Internet-Unternehmen wie Google und Microsoft angeschlossen. Es ist nicht das Ergebnis eines Interesseclearing zwischen Vertretern aller Beteiligten bzw. Betroffenen, sondern eine einseitig für maßgebend erklärte Setzung des mächtigeren Teils der Internetwirtschaft, die erhebliche Auswirkungen auf Dritte hat. Das Protokoll ist zwar auf dessen Akzeptanz durch den jeweiligen WebCrawler angewiesen; diese Akzeptanz liegt jedoch bei einem Großteil der marktbeherrschenden Internet-Konzerne vor.

Zwar gibt es jedenfalls für manche Märkte, insbesondere für den regionalen, wirtschaftlich starken Binnenmarkt der EU, auch differenzierte hoheitlich gesetzte Regeln. Für die auf globale IT-Aktivitäten ausgerichteten transnationalen und auch für viele nationale Märkte ist gleichwohl nur höchst begrenzt die hoheitlich gesetzte Rechtsordnung maßgebend. Vorherrschend sind die von der IT-Wirtschaft selbst organisierten Strukturen, die Art der Geschäftsverwirklichung und die dafür eigengesetzten Regeln.

\section{Schwierigkeiten hoheitlicher Verantwortungsübernahme}

Staatliche Akteure haben allerdings nach Maßgabe ihrer Rechtsordnung den Auftrag, im Rahmen ihrer Möglichkeiten Vorkehrungen zu treffen,

seine Folgen für die Netzwerkökonomie, 2018, 61 ff. (Der Beitrag enthält nicht nur viele Beispiele, sondern auch Anregungen für rechtliche Einhegungen selbstregulativer Ordnung insbes. der Plattformen); H. Schweitzer, Digitale Plattformen als private Gesetzgeber: Ein Perspektivwechsel für die europäische „Plattformregulierung", ZEuP 2019, $1 \mathrm{ff}$.

68 Zum REP s. A. Conrad/T. Schubert, How to Do Things with Code, GRUR 2018, 350 ff. S. a. T. Höppner, Das Verhältnis von Suchmaschinen zu Inhalteanbietern an der Schnittstelle von Urheber- und Kartellrecht, in: Wettbewerb in Recht und Praxis 2012, 625, $631 \mathrm{f} ., 636 \mathrm{ff}$. 
dass auch bei gesellschaftlicher Selbstgestaltung und Selbstregulierung Rechtsgüter respektiert und schützenswerte Interessen gewahrt werden. Der moderne, normativ durch Schutz- und Gewährleistungsaufgaben (s. o. VI) geprägte Staat als Gewährleistungsstaat ${ }^{69}$ unterliegt einer auf die Erfüllung entsprechender Aufträge ausgerichteten besonderen Gewährleistungsverantwortung. Speziell mit dem Blick auf die digitale Transformation der Gesellschaft ist staatlicherseits zu gewährleisten, dass durch rechtliche Rahmensetzung, Strukturvorgaben und inhaltliche Orientierungen sowie Verfahrenssicherungen, aber auch durch Flexibilitätsvorkehrungen die Funktionsfähigkeit und ggf. Anpassungsfähigkeit der Ordnungen nicht gefährdet wird und öffentliche Aufgaben auch bei ihrer Wahrnehmung durch Private unter Berücksichtigung von Gemeinwohlzielen erfüllt werden. Hier sind Vorkehrungen hoheitlich regulierter gesellschaftlicher Selbstgestaltung/ Selbstregulierung ${ }^{70}$ angezeigt, die insbesondere auf das jeweilige Potential an Chancen und Risiken abgestimmt sein müssen.

Die mit der digitalen Transformation verbundene privatwirtschaftlich verfasste Gesellschaftlichkeit im Internet hat allerdings zu einer weitgehenden Entkoppelung von den staatlicherseits gesetzten Strukturen geführt. Für die Art und Reichweite der privat gesetzten Weichenstellungen ist auch von Bedeutung, dass die von der Internetwirtschaft gesetzten Regeln nicht notwendig die Gestalt ausgearbeiteter Texte haben. Sie sind vielmehr in starkem Maße (nur) in die Technik selbst eingeschrieben worden. Dies geschieht etwa durch die Gestaltung der Funktionalität - wie der Filterung, Priorisierung und Darstellung von Informationen - oder sonstige Standardeinstellungen. In der Folge sind die praktizierten Regeln nur schwer erkennbar und kontrollierbar.

Auch insofern bleibt allerdings die staatliche Schutz- und Gewährleistungsverantwortung bestehen. ${ }^{71}$ Regulierungen ${ }^{72}$ können Vorkehrungen

$69 \mathrm{Zu}$ ihm allgemein G. F. Schuppert, The Ensuring State, in: A. Giddens (Hrsg.), The progressive manifesto. New ideas for the centre-left, 2003, $54 \mathrm{ff} . ;$ R. Ruge, Die Gewährleistungsverantwortung des Staates und der Regulatory State, 2004; C. Franzius, Gewährleistung im Recht: Grundlagen eines europäischen Regelungsmodells öffentlicher Dienstleistungen, 2009.

70 Ausführlicher dazu W. Hoffmann-Riem (Fn. 66).

71 Dazu s. auch S. Hindelang, Freiheit und Kommunikation, 2019, $237 \mathrm{ff}$., der die Schutzpflicht insbesondere zur Sicherung selbstbestimmter kommunikativer Teilhabe in der vernetzten Gesellschaft betont und deren Umsetzung im weiteren Verlauf seiner Untersuchung näher spezifiziert.

72 Auf die Vielfalt und Schwierigkeiten möglicher Regulierung kann ich hier nicht eingehen. In früheren Abhandlungen habe ich das eingehender getan, s. etwa $W$. Hoffmann-Riem (Fn. 5, 37, 66, 96,131). 
für die unter den Bedingungen der Digitalisierung wichtige Transparenz ${ }^{73}$ und Nachvollziehbarkeit der Vorgehensweisen ${ }^{74}$ treffen, darunter durch die Schaffung von Dokumentationspflichten, Begründungspflichten sowie Korrektur- und Kontrollvorgaben, ${ }^{75}$ gegebenenfalls erweitert durch Haftungsregimes. ${ }^{76}$ Auch können in Bezug auf die Inhalte und die Zugänglichkeit digitaler Dienste rechtliche Mindestanforderungen - so an die Art und Qualität der Leistungen und Produkte (etwa Diskriminierungsverbo$\mathrm{te}^{77}$ ) - geschaffen werden, die im Zuge einer Zertifizierung auch proaktiv überprüft werden können.

Die Normierung von Pflichten zu proaktiven und reaktiven Folgenabschätzungen sind weitere Möglichkeiten. Wichtig sind auch bereichsspezifische Vorkehrungen zur Korrektur von unerwünschten Entwicklungen. Innovationsermöglichungsrecht zielt aber auch darauf, erwünschte Innovationen zu stimulieren - etwa durch Anreize, aber ebenfalls durch die Vorsorge für Strukturen, die auch kleineren Unternehmen und Start-ups innovative Entwicklungen ermöglichen. Letztere aber sind bei Erfolg der Versuchung ausgesetzt, die häufig finanziell sehr attraktiven Angebote marktstarker Unternehmen zu ihrer Übernahme anzunehmen. Als Gegengewicht könnte eine branchenspezifisch ausgestaltete, die üblichen Aufgreifkriterien für Fusionen herabsetzende und damit eine wirksamere Fusionskontrolle als bisher eingerichtet werden. An dieser oder anderen wirksamen Vorkehrungen aber fehlt es.

Allerdings befinden sich Hoheitsträger gelegentlich in einer Dilemmasituation. Dies sei an einem Beispiel aus dem Bereich der Internetkommunikation illustriert. Dieses Beispiel gilt dem Versuch, ehrverletzende, gewalt-

$73 \mathrm{Zu}$ unterschiedlichen Dimensionen von Transparenzforderungen s. M. Martini (Fn. 5), $176 \mathrm{ff}$.

74 Zur Problematik vertiefend T. Wischmeyer, Regulierung intelligenter Systeme, AöR 143 (2018), 1, 18 ff., 42 ff.

75 Beispiele dafür - wenn auch dort nur zum Datenschutz - in: Gutachten der Datenethikkommission, 2019, Nr. 38. Entsprechende Regelungen gibt es aber auch außerhalb des Datenschutzes i. e. S.

$76 \mathrm{Zu}$ letzteren s. beispielsweise H. Zech (Fn. 1) sowie Ders., Entscheidungen digitaler autonomer Systeme: Empfehlen sich Regelungen zu Verantwortung und Haftung? Gutachten A 73. DJT, 2020; C. D. Müller-Hengstenberg/S.Kirn, Rechtliche Risiken autonomer und vernetzter Systeme, 2016, 163 ff.; G. Spindler, Haftungsrecht im Zeitalter des Internet of Things - von selbststeuernden Autos und denkenden Kühlschränken, Juristische Studiengesellschaft, Jahresband 2018, 125 ff.; D. Wielsch, Funktion und Verantwortung. Zur Haftung im Netzwerk, Rechtswissenschaft 2019, $84 \mathrm{ff}$.

77 Zu Diskriminierungspotentialen s. statt vieler M. Martini (Fn. 5), 47 ff., $334 \mathrm{ff}$. 
verherrlichende, pornographische oder als Fake-News eingeordnete Inhalte im Internet zu unterbinden. Hoheitliche Eingriffe zur Entfernung solcher Inhalte oder auch nur die Erschwerung des Zugangs zu ihnen geraten leicht in die Nähe der verfassungsrechtlich verbotenen staatlichen Zensur (Art. 5 Abs. 1 Satz 3 GG). ${ }^{78}$ Deshalb wird ein Vorgehen durch die Informationsintermediäre (etwa Facebook oder Google) vielfach als vorzugswürdig angesehen. Der EuGH hat in seiner Google-Entscheidung ${ }^{79}$ diesen Weg gewählt. Sie läuft darauf hinaus, dass die Internetunternehmen entscheiden, welche Inhalte im Internet akzeptabel bzw. nicht akzeptabel sind.$^{80}$ Damit kuratieren und korrigieren sie - wie Dolata ${ }^{81}$ formuliert hat (s. o. VII. 2) Inhalte, Informationsflüsse und Diskussionen. Für eine angemessene Lösung des Problems bedarf es einer stärkeren Berücksichtigung des Umstandes, dass die Kommunikationsintermediäre durch ihre Entscheidungen über die Bedingungen der Verwirklichung von Freiheitsrechten der Betroffenen - sowohl der Äußerer als auch der von einer Äußerung negativ Betroffenen - und zugleich über Inhalte öffentlicher Diskurse entscheiden und damit auch eigene Diskursmacht ausüben. Hier bedarf es eines den verschiedenen Informationszugangsinteressen gerecht werdenden Ausgleichs.

Die Maßstäbe und das Verfahren näher zu bestimmen, ist eine hoheitliche Aufgabe zur Gewährleistung der Freiheitsverwirklichung (s. o. VI) in einer den Besonderheiten von öffentlichen Kommunikationsnetzwerken gerecht werdenden multipolaren Konfliktkonstellation. Diese Aufgabe kann nicht allein den nicht demokratisch legitimierten Intermediären zur Selbstgestaltung übertragen werden. Hoheitsträger - je nach Regelungskompetenz etwa die EU oder die einzelnen Staaten - sind aufgefordert, für die Wahrnehmung der Verantwortung durch die Intermediäre einen auch rechtsstaatliche Anforderungen wahrenden regulativen Rahmen zu schaffen (darunter Anhörungsrechte und Rechtsbehelfe sowie Aufsichtsvorkeh-

78 Zur Problematik s. etwa die Diskussion um das deutsche Netzwerkdurchsetzungsgesetz. Dazu s. M. Eifert/T. Gostomzyk (Hrsg.) Netzwerkrecht. Zur Zukunft des NetzDG und seine Folgen für die Netzwerkökonomie, 2018; I. Löber/A. Roßnagel, Das Netzwerkdurchsetzungsgesetz in der Umsetzung, MMR 2019, 71. Für 2020 ist eine Novellierung geplant.

79 EuGH, Urteil vom 13.05.2014, Rs. C-131/12 (Google Spain), EuGRZ 2014, 320 ff.

$80 \mathrm{Zu}$ den grundrechtlichen Fragen, wieweit die Betreiber sozialer Medien zur Löschung von Inhalten ihrer Nutzer berechtig sind, s. J. Lüdemann, Grundrechtliche Vorgaben für die Löschung von Beiträgen in sozialen Netzwerken, MMR 2029, $279 \mathrm{ff}$.

81 U. Dolata (Fn. 64). 
rungen). Staatliche und private Verantwortung müssen miteinander verwoben werden. ${ }^{8283}$

Hoheitliche Regulierung im Digitalbereich steht nicht nur hier vor der Schwierigkeit, auf die Begleiterscheinungen und Folgen der Digitalisierung regulativ angemessene Lösungen zu finden. Ein besonderes Problem ist dabei die begrenzte Vorhersehbarkeit vieler Entwicklungen und damit auch neuer Regelungsbedarfe, aber auch der Wirksamkeit hoheitlicher Interventionen. Soll Recht auch im IT-Bereich als chancen- und risikoorientiertes Innovationsermöglichungsrecht konzipiert sein (s. o. V), ist mit der Schwierigkeit umzugehen, die Entstehung und Anwendung des Neuen regulativ umhegen zu sollen, obwohl das Neue selbst oder die von ihm ausgehenden Folgen, insbesondere die Risiken, nicht oder nur begrenzt bekannt sind.$^{84} \mathrm{Zu}$ bewältigen ist das Problem vorhersehbarer Unvorhersehbarkeit. ${ }^{85}$ Das ist zwar keine Besonderheit des IT-Bereichs, bedarf aber hier eines auf die gegenwärtige Transformation abgestimmten Vorgehens.

\section{Herausforderungen an bereichsspezifisches Recht hinsichtlich des Einsatzes digitaler Techniken}

\section{Ausweitung rechtlicher Aufmerksamkeit}

Da die digitale Transformation fast alle gesellschaftlichen Bereiche erfasst hat, bedeutet sie eine Herausforderung für das Rechtssystem in seinen verschiedenen Gegenstandsbereichen und auf seinen verschiedenen Ebenen. Die gesamte Rechtsordnung muss daher daraufhin besehen werden, ob und wieweit sie angemessen auf die durch die Digitalisierung bedingten Disruptionen und Transformationen reagiert.

$82 \mathrm{Zu}$ den Schwierigkeiten und Möglichkeiten s. die Analyse von D. Wielsch, Funktion und Verantwortung. Zur Haftung im Netzwerk, Rechtswissenschaft 2019, 84 ff. S. auch Ders. (Fn. 67).

83 Ein Versuch zur rechtlichen Regelung ist das deutsche Netzwerkdurchsetzungsgesetz, das aber seinerseits sehr umstritten ist. Zu diesem Gesetz s. statt vieler F. Kalscheuer/C. Hornung, Das Netzwerkdurchsetzungsgesetz - Ein verfassungswidriger Schnellschuss, NVwZ 2017, 1721. S. ferner die Nachw. in Fn. 78.

84 Dazu s. D. Sauer, Perspektiven sozialwissenschaftlicher Innovationsforschung eine Einleitung, in: D. Sauer/C. Lang (Hrsg.), Paradoxien der Innovation. Perspektiven sozialwissenschaftlicher Innovationsforschung, 1999, 9, 14.

85 Dazu s. Hase, Die Komplexität des Wissens und die Legitimation rechtlicher Normsetzung, in: B. Buchner/K.-H. Ladeur (Hrsg.), Wissensgenerierung und -verarbeitung im Gesundheits- und Sozialrecht, 2016, 125, $134 \mathrm{f}$. 
Da die Anwendbarkeit von Recht nicht, jedenfalls soweit dies nicht ausdrücklich vorgesehen ist, auf überkommene (etwa analoge) Vorgehensweisen begrenzt ist, liegt es zunächst nahe, von seiner Geltungskraft auch bei der Nutzung digitaler Technologien in den jeweils betroffenen gesellschaftlichen Bereichen auszugehen. Dass eine simple Analogie dafür aber vielfach nicht taugt, hat Herbert Zech in seinem Referat dargelegt. ${ }^{86}$ Soweit Unterschiede zwischen dem Einsatz und den Folgen traditioneller und digitaler Techniken bestehen, lässt sich dies allerdings zumindest teilweise durch veränderte Auslegung bestehenden Rechts berücksichtigen. Dies gilt auch für die Reichweite der in der Verfassung vorgesehenen Verbürgungen, etwa der Grundrechte, ${ }^{87}$ aber ebenso für die sonstige Rechtsordnung.

Es bleiben angesichts der Besonderheiten von Einsatzmöglichkeiten und Wirkungen der Digitalisierung aber Bedarfe für Sonderregeln, die zum Teil in übergreifend geltenden Gesetzen wie dem BGB, StGB, UrhG, GWB und UWG vorgesehen werden können, häufig aber auch in bereichsbezogenen Normierungen einfachen Rechts - wie Medizinrecht, Finanzmarktrecht, Telemedienrecht, Rundfunk- und Telekommunikationsrecht - enthalten sind oder dort erfolgen sollten. Dies kann sowohl der Verbesserung der Chancen als auch der Minimierung und Abwehr von Risiken dienen, aber auch Vorkehrungen zur Korrektur von Fehlentwicklungen umfassen.

Besondere Regelungen sind beispielsweise angezeigt als Vorsorge gegenüber der Verwirklichung von Risiken beim Einsatz digitaler Techniken etwa in der Forschung und für die Nutzung ihrer Ergebnisse im Zuge deren Anwendung. Dies betrifft etwa den Gesundheitsbereich oder den Umgang mit der Gentechnologie. Regelungsbedarf besteht auch für das automatisierte Fahren im Straßenverkehr. Gleiches gilt für das Arbeitsschutzrecht oder das Kapitalmarktrecht. ${ }^{88}$ Bereichsspezifische Regelungen gibt es in solchen Feldern schon; sie müssen aber in Vielem neu auf die Folgen der Digitalisierung abgestimmt werden. Dabei ist auch zu klären, welche der

\section{I. d. B.}

87 Hierfür steht beispielhaft die Rechtsprechung des Bundesverfassungsgerichts zum grundrechtlichen Schutz im Bereich der Digitalisierung seit der Entwicklung des Grundrechts auf informationelle Selbstbestimmung. S. zu ihr W. Hoffmann-Riem (Fn. 48), 569 ff. S. auch C. Hoffmann/A. D. Luch/S. Schulz/K. C. Borchers, Die digitale Dimension der Grundrechte, 2015.

$88 \mathrm{Zu}$ bereichsspezifischen Analysen mit dem besonderen Blick auf den Einsatz von KI s. etwa die Beiträge in T. Wischmeyer/T. Rademacher (Hrsg.), (Fn. 5), Part 2. Ebenso bereichsspezifische Analysen mit dem Blick auf Big Data enthalten die Beiträge in T. Hoeren (Hrsg.) (Fn. 34). 
in das Tätigkeitsfeld bzw. in die Wertschöpfungskette einbezogenen Akteure aufgrund ihres Wissens und von Möglichkeiten der Einwirkung auf die Vermeidung von Risiken in Anspruch genommen werden sollen.

\section{Normwandel durch transformative Veränderung des Realbereichs von Normen}

Die digitale Transformation lässt die Rechtsordnung auch dort nicht unberührt, wo der Wortlaut der Gesetze unverändert bleibt. Insoweit ist insbesondere auf die Relevanz des Realbereichs von Normen zu verweisen. Gemeint sind die in der Norm - sei es explizit oder implizit - in Bezug genommenen technologischen, naturwissenschaftlichen, sozialen, politischen, ökonomischen, kulturellen, ökologischen u. ä. Realitäten in ihren Grundstrukturen. ${ }^{89}$ Dieser Ausschnitt der nichtrechtlichen Wirklichkeit ist ein konstitutiver Bestandteil der Norm, der neben deren Sprach- bzw. Textbereich und der systematischen Einbettung der Norm in die Gesamtrechtsordnung für den normativen Gehalt bedeutsam ist. ${ }^{90}$ Angesichts der Ubiquität der transformativen Informationstechnologie und ihrer innovativen Potentiale erhalten die Inhalte informationstechnisch relevanter Normen infolge der im IT-Bereich häufigen Realbereichsänderungen eine transformative Dynamik. ${ }^{91}$ Vergleichbares gilt auch für im IT-Bereich nutzbare soziale sowie ethische Normen.

Zwar kann bekanntlich aus dem Sein nicht auf das Sollen geschlossen werden. Da aber der Realbereich von Normen einer der Bezugspunkte zur Inhaltsdeutung von Normen ist, wirken Änderungen in ihm - also im Sein - auf das rechtlich bestimmte Dürfen, Können und gegebenenfalls Sollen ein. ${ }^{92}$ Je schneller der technologische Fortschritt und seine Auswirkungen in den gesellschaftlichen Handlungsfeldern erfolgen, umso häufiger und nachhaltiger kann es infolge des Wandels des Realbereichs zum Normwandel auch ohne Eingreifen des Gesetzgebers kommen.

89 W. Hoffmann-Riem (Fn. 48), 113.

90 Vgl. K. Hesse, Grundzüge des Verfassungsrechts der Bundesrepublik Deutschland, 20. Aufl. 1999, Rn. 45 f., F. Müller/R. Christensen, Juristische Methodik. Bd. I, 11. Aufl., 2013.

91 S. dazu B. Fateh-Moghadam, Innovationsverantwortung im Strafrecht: Zwischen Strict Liability, Fahrlässigkeit und erlaubtem Risiko - zugleich ein Beitrag zur Digitalisierung des Strafrechts, ZSTW 2019, 113 (4), 863, $872 \mathrm{ff}$.

92 B. Fateh-Moghadam (Fn. 91), 867. 


\section{Zur begrenzten Reichweite des bisherigen Datenschutzrechts}

Für die von der Digitalisierung betroffenen Bereiche hat selbstverständlich das auf Persönlichkeitsschutz gerichtete traditionelle Datenschutzrecht weiter Bedeutung. Es wäre aber verfehlt, die Bemühungen um rechtliche Regelungen beim Einsatz digitaler Techniken auf den Schutz personenbezogener Daten zu konzentrieren und damit von der Vielfalt der in der Verarbeitung von Daten unterschiedlicher Art betroffenen Rechtsgüter und der Notwendigkeit ihres Schutzes abzulenken. ${ }^{93}$

Die bisher typische Konzentration der Sichtweise vor allem in der deutschen Öffentlichkeit auf Datenschutzrecht entstand in der zweiten Hälfte des vorigen Jahrhunderts parallel zum Ausbau der Leistungsfähigkeit von Computern. Dies war unter anderem begleitet von Befürchtungen vor Ausspähungen oder Ausforschungen durch staatliche Organe und private Akteure. Die Fokussierung auf Datenschutz wurde in Deutschland auch durch öffentlich stark beachtete Gerichtsentscheidungen gestützt, etwa durch die verfassungsgerichtlichen Entscheidungen zu dem aus der Garantie der Menschenwürde (Art. 1 Abs. 1 GG) und dem Persönlichkeitsrecht (Art. 2 Abs. 1 GG) abgeleiteten Schutz informationeller Selbstbestimmung, dies insbesondere zwecks Begrenzung staatlicher Überwachung. ${ }^{94}$

Der schnelle Aufstieg sozialer Netzwerke und die große Zahl dabei anfallender, insbesondere personenbezogener, Daten und deren Verwertung steigerten die Konzentration der Aufmerksamkeit auf dieses Feld, mit der Folge der Schaffung auch darauf bezogener nationaler Datenschutzgesetze und Datenschutzbehörden, aber auch europäischer Regelungen, wie etwa der EU-Datenschutzgrundverordnung (DSGVO) und weiterer europarechtlicher Bestimmungen zum Datenschutz. ${ }^{95}$ So hat die DSGVO eine Fülle von Schutzvorkehrungen getroffen, darunter Datenschutzgrundsätze, Zweckbegrenzungen und Zulässigkeitsregeln. Hinzu kommen Infor-

93 S. zur Notwendigkeit der Ausweitung des Blicks beispielsweise R. Broemel/H.-H. Trute, Alles nur Datenschutz? Zur rechtlichen Regulierung algorithmenbasierter Wissensgenerierung, Berliner Debatte Initial 27 (2016), 50 ff.

94 S. zu dieser Rechtsprechung die auch weitere Grundrechte einbeziehende Darstellung in W. Hoffmann-Riem, (Fn. 48), $569 \mathrm{ff}$.

95 So zielt beispielsweise die noch geltende E-Privacy Richtlinie (Richtlinie 2002/58/EG des Europäischen Parlaments und des Rates vom 12. Juli 2002) sowie die als deren Ersatz geplante, aber noch im Erlassverfahren befindliche E-PrivacyVerordnung der EU auf personenbezogenen Datenschutz speziell im Bereich der elektronischen Kommunikation ab und soll den freien Verkehr der Daten und von elektronischen Kommunikationsgeräten und -diensten in der Gemeinschaft gewährleisten. 
mations- und Auskunftsrechte und -pflichten sowie vor allem das als Regelfall vorgesehene Erfordernis der Einwilligung der Betroffenen in die Erhebung und Verwertung personenbezogener Daten.

In der Praxis sind der Regelungen der DSGVO allerdings nur begrenzt wirksam. So kennt die DSGVO viele Ausnahmen und sie verzichtet auf Differenzierungen je nach den betroffenen Akteuren. So gelten die normierten Datenschutzgrundsätze, Zulässigkeitsregeln, Zweckbegrenzungen oder Rechte der betroffenen Personen für private Vereine oder kleine Unternehmen ebenso wie für die Plattformen der Internetkonzerne. Eine Folge dessen ist, dass alle Nutzer digitaler Angebote mit einer Vielzahl, Vielfalt und meist großer Länge von ihnen schwer verständlichen Regeln konfrontiert sind - mit der hohen Wahrscheinlichkeit der Abstumpfung beim Umgang mit ihnen.

Vor allem aber versagt das als zentrales Schutzmittel gepriesene Einwilligungserfordernis als effektives Mittel zum Autonomieschutz der Nutzer in vielerlei Hinsicht. ${ }^{96}$ Seine Wirksamkeit wird vor allem dadurch ausgehebelt, dass die Unternehmen die Nutzung ihrer Dienste in den einseitig gesetzten Geschäftsbedingungen davon abhängig machen, dass zuvor in die Erhebung und Verwertung von Daten vielfach auch insoweit eingewilligt wird, als diese für die Erbringung des nachgefragten Dienstes gar nicht erforderlich sind ${ }^{97}$ So werden in den Geschäftsbedingungen der Unternehmen meist sehr großzügig Erhebungs- und Verwertungszwecke festgelegt, auf die sich die Einwilligung erstrecken soll, darunter insbesondere auch solche, die für Nutzer selbst keinen erkennbaren Vorteil bringen. Aber auch dort, wo in den AGBs behauptet wird, die Verwertung diene der Verbesserung der Leistung für die Nutzer, wird nicht spezifiziert, ob und in-

96 Aus der reichhaltigen Literatur zur Leistungskraft des Einwilligungserfordernisses vgl. etwa Y. Hermstrüwer, Informationelle Selbstgefährdung, 2016, m. w. Hinw.; P. Radlanski, Das Konzept der Einwilligung in der datenschutzrechtlichen Realität, 2016; W. Hoffmann-Riem, Verhaltenssteuerung durch Algorithmen - Eine Herausforderung für das Recht, AöR 2017, 1, 20 ff.; S. Strauß, Dashcam und Datenschutz, NZV 2018, 554; P. Uecker, Die Einwilligung im Datenschutzrecht und ihre Alternativen, ZD 2019, 248. Zur Frage, ob für die Einwilligung in die Nutzung von Cookies eine opt-in Kontruktion gefordert ist s. EuGH Rs. C-637/17, EuGRZ 2019, 486 sowie BGH I ZR 7/16 vom 28.5.2020 (jeweils bejahend).

97 Art. 7 i. V. m. Art. 4 Nr. 11 DSGVO gewährt insofern zwar Schutz; welcher aber nur begrenzt wirksam ist. Diese Norm verlangt zwar die Freiwilligkeit der Einwilligung, Art. 7 Abs. 4 ist aber nicht als striktes Koppelungsverbot formuliert und wird in der Praxis nur als Berücksichtigungspflicht verstanden; so auch J. H. Klement, in: S. Simits/G. Hornung/I. Spieker genannt Döhmann, Datenschutzrecht 2019, Rn. 58 zu Art. 7 DSGVO mit Nachw. auch zu Gegenmeinungen in Fn. 111. 
wiefern es für diesen Maßstab auch auf die Sichtweise der Nutzer ankommt. Von den Unternehmen selbst wird insbesondere die Möglichkeit der Personalisierung der Angebote als Verbesserung ihrer Informationsstrategie eingestuft, die es ihnen ermöglicht, eine gezielte Selektion der zugänglichen Inhalte nach Maßgabe der Auswertung früher erhobener Daten oder der Zuordnung zu Clustern von Personen mit bestimmten Werthaltungen, Verhaltensweisen und Aufmerksamkeiten u. a. durchzuführen. Dies kann bewirken, die Betroffenen tendenziell in einer kommunikativen Komfortzone zu halten, bestimmte Werthaltungen zu verfestigen, irritierende Erfahrungen vorzuenthalten ${ }^{98}$ und jedenfalls bemüht zu sein, nicht die Aufmerksamkeit der Nutzer - aus der Sicht der Unternehmen ein besonders wichtiges Gut - zu verlieren. ${ }^{99}$

Die Nutzung der Daten zu den in den AGBs meist großzügig umschriebenen Zwecken erlaubt den Unternehmen die Erwirtschaftung des von Shoshana Zuboff analysierten, lukrativ verwertbaren Verhaltensüberschusses bzw. des von Armin Nassehi so genannten Sinnüberschusses (s. o. II.2). Soweit die Bürgerinnen und Bürger im privaten oder beruflichen Leben auf die Nutzung digitaler Dienste angewiesen sind oder sich nicht kommunikativ isolieren wollen, bleibt ihnen praktisch keine Möglichkeit, die Einwilligung zu verweigern, jedenfalls soweit es - wie regelmäßig, - keine alternativen Angebote für Dienste gleicher Qualität und Verbreitung sowie ohne Abschöpfung des „Verhaltensüberschusses“ gibt.

Diese Problemlage ist auch dem BVerfG bewusst, wie die folgende Umschreibung zeigt: „In allen Lebensbereichen werden zunehmend für die Allgemeinheit grundlegende Dienstleistungen auf der Grundlage umfänglicher personenbezogener Datensammlungen und Maßnahmen der Datenverarbeitung von privaten, oftmals marktmächtigen Unternehmen erbracht, die maßgeblich über die öffentliche Meinungsbildung, die Zuteilung und Versagung von Chancen, die Teilhabe am sozialen Leben oder auch elementare Verrichtungen des täglichen Lebens entscheiden. Die einzelne Person kommt kaum umhin, in großem Umfang personenbezogene Daten gegenüber Unternehmen preiszugeben, wenn sie nicht von diesen grundlegenden Dienstleistungen ausgeschlossen sein will. Angesichts der Manipulierbarkeit, Reproduzierbarkeit und zeitlich wie örtlich praktisch unbegrenzten Verbreitungsmöglichkeit der Daten sowie ihrer unvorher-

$98 \mathrm{Zu}$ dieser - häufig unter dem Stichwort Bubbleeffekt diskutierten - Situation s. E. Pariser, The Filter Bubble. What the Internet Is Hiding From You, 2011.

99 Zum knappen Gut „Aufmerksamkeit“ und die Bemühungen, sie zu sichern, vgl. G. Franck, Ökonomie der Aufmerksamkeit, 1998. 
sehbaren Rekombinierbarkeit in intransparenten Verarbeitungsprozessen mittels nicht nachvollziehbarer Algorithmen können die Einzelnen hierdurch in weitreichende Abhängigkeiten geraten oder ausweglosen Vertragsbedingungen ausgesetzt sein.“ 100

\section{Zur gestiegenen Bedeutung nicht personenbezogener Daten}

Die Ausführungen zur begrenzten Bedeutung überkommenen Datenschutzrechts dürfen nicht dahin gedeutet werden, es sei als solches obsolet. Für den Umgang mit den Folgen der digitalen Transformation muss aber der Befund berücksichtigt werden, dass diese Datenschutznormen nur einen begrenzten Wirkungsbereich haben.

Das traditionelle Datenschutzrecht ist auch dann nicht anwendbar, wenn nicht personenbezogene Daten die Grundlage für personenbezogene Aktivitäten bilden, etwa für das Targeting, eine Form der Informationssteuerung durch eine auf Zielgruppen maßgeschneiderte Ansprache. Solche Zielgruppen können auch ohne vorherige Erfassung personenbezogener Daten der konkret betroffenen Personen gebildet werden, insbesondere unter Nutzung von durch Mustererkennung gebildeten Clustern, also typischen Gruppen von Personen, auf die gemeinsame Beschreibungsmerkmale zutreffen. Solche Cluster können - auch wenn sie nicht auf individuelle Personen bezogen sind - zur gezielten Einwirkung auf Personen genutzt werden, die aufgrund anderer, nicht vom Datenschutzrecht erfasster Informationen mit Hilfe von Datenkombinationen dem Cluster zugerechnet werden. Durch solche clusterbezogenen Einwirkungen können ebenso Werthaltungen, Erfahrungswissen oder das Verhalten bei politischen Wahlen beeinflusst werden wie bei der Verwendung personenbezogener Daten für solche Beeinflussungen. Auch können auf der Grundlage diverser Daten Trendanalysen und Prognosen erstellt werden, die für unterschiedliche politische oder geschäftliche Strategien einsetzbar sind, dabei auch für die Verhaltensbeeinflussung von Personen, deren persönliche Daten nicht für solche Zukunftsaussagen herangezogen worden waren.

Besonders wichtig ist, dass das herkömmliche Datenschutzrecht keinen Schutz gegenüber der Erhebung und Verwertung anderer als personenbe-

100 In BVerfG NJW 2020, 300, Rn. 85 (die Entscheidung bezog sich auf das Grundrecht auf informationelle Selbstbestimmung). 
zogener Daten gewährt. ${ }^{101}$ Auch Daten ohne diesen Personenbezug sind auf vielfältige Weise nutzbar und werden gegenwärtig für Wirtschaft, Technologie, Technikentwicklung und Politik u. a. immer bedeutsamer. Nicht zufällig sind in der EU eigenständige Regelungen für solche sonstigen Daten geschaffen worden, wenn auch nicht allgemein, sondern speziell zur Sicherung der Wirkungsweise des Binnenmarktes durch Abbau von Hindernissen für die Datenmobilität und die Anbieterabhängigkeit. Verwiesen sei auf die darauf bezogene EU-Verordnung ${ }^{102}$ und ergänzende „Leitlinien“. 103

Nicht als personenbezogene Daten sind Daten einzuordnen, die durch Anonymisierung und Vorkehrungen gegen eine Deanonymisierung nicht mehr bestimmten Personen zugeordnet werden können. Gleiches gilt für aus dem Verhalten von Personen generierte Daten ohne Persönlichkeitsrechtsbezug. Nicht in den Geltungsbereich des Datenschutzrechts fallen ferner durch Datenverarbeitung gewonnene neue Daten ohne Personenbezug (insbesondere Datenderivate), ebenso die eines Personenbezugs entkleideten aggregierten Daten und selbstverständlich rein industrielle Daten, ${ }^{104}$ etwa solche, die bei der Produktion von Gütern oder bei deren Vertrieb erhoben werden. Auch gibt es so genannte synthetische Daten, ${ }^{105}$ das heißt den Ausgangsdatensätzen in ihren statistischen Informationen und Strukturen äquivalente Datensätze, deren Daten definitiv nicht auf die Ausgangswerte zurückgeführt werden können. Verfügbar können ebenfalls sogenannte offene Daten (Open Data) sein. ${ }^{106}$ Dazu zählen auch Da-

101 Personenbezogen sind Daten, die Angaben über persönliche oder sachliche Verhältnisse einer bestimmten Person enthalten oder erschließen lassen. Zur Definition s. Art. 4 Nr. 1 DSGVO.

102 Verordnung (EU) 2018/1807 des Europäischen Parlaments und des Rates vom 14. Nov. über einen Rahmen für den freien Verkehr nicht-personenbezogener Daten in der Europäischen Union.

103 Mitteilung der Kommission an das europäische Parlament und den Rat vom 29.05.2019, COM (2019) 250 final über „Leitlinien zur Verordnung über einen Rahmen für den freien Verkehr nicht-personenbezogener Daten in der Europäischen Union".

104 Dazu s. statt vieler A. Wiebe/N. Schur, Das Recht an industriellen Daten im verfassungsrechtlichen Spannungsverhältnis zwischen Eigentumsschutz, Wettbewerbs- und Informationsfreiheit, ZUM 2007, 461.

105 Dazu s. https://www.stiftung-nv.de/sites/default/files/synthetische_daten.pdf.in.

106 Dazu s. die Richtlinie (EU) 2019/1024 des Europäischen Parlaments und des Rates vom 20. Juni 2019 über offene Daten und die Weiterverwendung von Informationen des öffentlichen Sektors. 
ten aus dem Bereich der öffentlichen Verwaltung, ${ }^{107}$ insbesondere solche, die über die Inanspruchnahme der Möglichkeiten der Informationsfreiheitsgesetze zugänglich werden. Schließlich gibt es auch von Privaten bereitgestellte offene Daten. Zu erwähnen sind ferner Daten, die von mehreren geteilt werden und insbesondere über Märkte zum Data Sharing zugänglich sind. ${ }^{108}$

In vielen Praxisbereichen fallen personenbezogene, vielfach aber weitgehend nur nicht personenbezogene Daten an. Sie alle können insbesondere durch Dekontextualisierung zur Bildung eines Pools für Daten beitragen, die unterschiedlichen Kombinationen zugänglich und für unterschiedliche Zwecke nutzbar sind. ${ }^{109}$ In den meisten Feldern der Anwendung digitaler Techniken in der Industrie, in der Forschung, im Handelsverkehr oder in Kapitalmärkten überwiegt die Nutzbarkeit nichtpersonenbezogener Daten.

Gerade die durch Kombination verschiedener Daten gewonnenen Erkenntnisse sind eine wesentliche Grundlage von neuem Wissen und des auf dessen Verwendung aufbauenden wirtschaftlichen Erfolgs von IT-Unternehmen sowie von anderen Unternehmen, die digitale Techniken für Analysen, Prognosen, Beratungen, Produktionsentscheidungen, Geschäftsstrategien u.a. nutzen. Solches Wissen kann ebenfalls für staatliche Stellen wichtig sein.

Daten verschiedenster Art werden als „Rohstoffe“ für die Erzeugung von Wissen auch zwecks Erfüllung von Gemeinwohlbelangen verwendet. Ein aktuelles Beispiel ist der Gesundheitssektor. Die Transformation der Wissenserzeugung im Gesundheitssektor und der damit verbundene Übergang von einer erfahrungsgeleiteten zu einer evidenzbasierten Medizin war ein Thema in Thomas Vestings Referat, ${ }^{110}$ auf das ich verweise. Da die Gesellschaft und ihre Mitglieder ein Interesse an einem leistungsfähigen

107 Als Beispiel s. die Übersicht in: Bertelsmann Stiftung, Musterkatalog für Kommunen. Welche offenen Daten werden von Kommunen Nordrhein-Westfalens veröffentlicht, 2020.

$108 \mathrm{Zu}$ den Interessen an und Möglichkeiten für Data Sharing s. H. Richter/P. R. Slowinski, The Data Sharing Economy: On the Emergence of New Intermediaries, IIC 2019, 4 ff.

109 Die Arten und Vielfalt von anfallenden Daten illustriert die - auf das automatisierte vernetze Kfz-Fahren bezogene - Auflistung bei G. Hornung, Ökonomische Verwertung und informationelle Selbstbestimmung, in: A. Roßnagel/G. Hornung, (Hrsg.), Grundrechtsschutz im Smart Car, 2019, S. 112. Zum rechtlichen Umgang mit und dem wirtschaftlichen Wert von Automobildaten s. ders./T. Goeble, „Data Ownership“ im vernetzten Automobil, CR 2015, 265 ff.

110 I. d. B. 
Gesundheitswesen haben, sind die Wissenserzeugungsprozesse im Gesundheitssektor von öffentlicher Bedeutung. ${ }^{111}$ Daher ist es beispielweise ein aktuell brennendes Thema, ob es eine gesetzliche Pflicht zu einem Data Sharing auch im Hinblick auf personenbezogene Daten geben sollte, um über hinreichendes Datenmaterial verfügen zu können, das komplexe Auswertungen und die Entwicklung darauf aufbauender Diagnose- und Therapieinstrumente ermöglicht. Hierauf hat Timo Rademacher in seinem Referat schon hingewiesen. ${ }^{112}$ Nutzbar für medizinische Forschung sind aber auch viele nicht oder nicht mehr personenbezogene Daten oder die Kombination personenbezogener und sonstiger Daten. Soweit hier Bedarf für Rechtsschutz im Hinblick auf Daten besteht, ist das Medizinrecht nicht als spezielles Datenschutzrecht zu verstehen, sondern als bereichsspezifisches Recht über die Erfüllung von Aufgaben, die u.a. auch unter Hinzuziehung von Daten unter Einschluss von personenbezogenen Daten erfolgt.

\section{Vom Datenschutzrecht zum Datenverwendungsrecht}

Digitale Techniken werden - wie schon mehrfach erwähnt - in fast allen Lebensbereichen eingesetzt. Dafür gibt es bereichsbezogenes Sonderrecht, etwa Gentechnikrecht, Gesundheitsrecht, Lebensmittelrecht, Kapitalmarktrecht, Energierecht, Chemikalienrecht u. a. Der Einsatz digitaler Techniken in den betreffenden Handlungsfeldern dient der Interessenverwirklichung und dem Rechtsschutz mit Hilfe von Instrumenten, die auf die jeweiligen Gegenstandsbereiche abgestimmt sind. Soweit diese auch Fragen des Datenschutzes betreffen, können das allgemeine dem Persönlichkeitsschutz dienende Datenschutzrecht und/oder ein für das jeweilige Gebiet speziell geschaffenes Datenschutzrecht maßgebend werden, das vielfach auch Regeln über den Umgang mit Daten ohne Personenbezug enthält. ${ }^{113}$

111 S. etwa H. C. Röhl, Unternehmenswissen, Demokratie und Digitalisierung, in: R. Broemel/A. Pilniok (Hrsg.), Die digitale Gesellschaft als Herausforderung für das Recht in der Demokratie, 2020, 95, 105.

112 I. d. B.

113 Zur Illustration der insoweit bestehenden Vielfalt sei verwiesen auf $\mathrm{T}$. Hoeren/U. Sieber/B. Holznagel (Hrsg.), Handbuch Multimediarecht. Rechtsfragen des elektronischen Geschäftsverkehrs, Stand Okt. 2019, das eine Vielzahl unterschiedlicher Rechtsgebiete einschließlich der dort maßgebenden datenschutzrechtlichen Normen behandelt. 
Beispiele für besondere Regeln zur Datenerhebung und -verwendung finden sich in den im Strafverfahrensrecht, Polizeirecht und Nachrichtendienstrecht enthaltenen Ermächtigungen insbesondere zur Strafverfolgung bzw. Gefahrenabwehr. ${ }^{114}$ Ein auf das Polizeirecht bezogenes, besonderes ausdifferenziertes (und vermutlich in der Praxis nicht leicht zu handhabendes) Regelwerk ist das i. J. 2020 novellierte Hamburgische „Gesetz über die Datenverarbeitung der Polizei“ (PolDVG). ${ }^{115}$ Es regelt einen Großteil von Befugnissen über die Datenverarbeitung einerseits in allgemeiner Hinsicht $(\$ \$ 10-15)$, andererseits im Hinblick auf besondere Befugnisse, wie etwa den Einsatz erkennungsdienstlicher Maßnahmen, Eingriffe in die Telekommunikation, die elektronische Aufenthaltsüberwachung und polizeiliche Beobachtung sowie Maßnahmen der Datenübermittlung an andere Stellen oder zur Rasterfahndung. Hier geht es um die Schaffung besonderer Instrumente der Gefahrenvorsorge und -abwehr sowie der Strafverfolgung unter Verwendung von Daten. Die Besonderheiten der Materie ließen es dem Landesgesetzgeber nicht als sinnvoll erscheinen, nur auf die allgemeinen Grundsätze des Datenschutzrechts zu verweisen; vielmehr sollten spezifische Ermächtigungen mit der Möglichkeit der ausdifferenzierten Abwägung von Belangen des Persönlichkeitsschutzes einerseits und der Gefahrenabwehr und Strafverfolgung andererseits geschaffen werden.

Während dieses Gesetz inhaltlich den Schutz personenbezogener Daten einbezieht, gibt es eine Reihe von Gesetzen, die ausschließlich oder vorrangig Regelungen über Daten anderer Art enthalten. Dies legt eine Überprüfung nahe, ob und wie die Erfassung und der Umgang mit solchen Daten rechtlich geregelt wird. ${ }^{116}$

Dazu gehören das Urheberrecht und Patentrecht. Auf Möglichkeiten ihres Einsatzes und denkbarer Änderungen ist Linda Kuschel in ihrem Referat eingegangen. ${ }^{117}$ Auf ihre differenzierenden Ausführungen möchte ich statt einer eigenen Analyse verweisen.

114 Dazu s. statt vieler M. Bäcker/E. Denninger/K. Graulich (Hrsg.), Handbuch des Polizeirechts. Gefahrenabwehr - Strafverfolgung - Rechtsschutz, 6. Aufl. 2018, dort insbes. Kap. G und $\mathrm{H}$.

115 Gesetz vom 12.12.2019, HmbGVBl, 485. Zu ihm s. E. Richter, Polizei- und Ordnungsrecht, in: W. Hoffmann-Riem/H.-J. Koch (Hrsg.) Landesrecht Hamburg. Staats- und Verwaltungsrecht, 2020 (i. E).

116 Dazu s. L. Specht, Das Verhältnis möglicher Datenrechte zum Datenschutzrecht, GRUR Int. 2017, 1040 ff. Verwiesen sei auch auf die die Beiträge in M. Ebers et al (Fn. 5), die in den 31 Kapiteln dieses Rechtshandbuchs auch sehr ausführlich Literaturangaben enthalten.

117 I. d. B. 
Erwähnen möchte ich aber, dass viel darüber diskutiert wird, den Schutz für algorithmische Systeme und digitale Verwendungen auszubauen. ${ }^{118}$ Eine der vielen im Ergebnis weiter umstrittenen Frage lautet etwa, ob und wieweit es ein allgemeines Datenproduzentenrecht geben soll, insbesondere ein eigentumsähnlich ausgestaltetes Recht an industriellen und anderen im Wirtschaftsbereich wichtigen Daten. ${ }^{119}$ Dessen Anerkennung wäre in Anbetracht der großen Reichweite der digitalen Transformation auch ein Schritt zur verstärkten Ökonomisierung des Datenrechts.

Auch gibt es Diskussionen über ein Recht auf Zugang Dritter nicht nur zu staatlich verfügbaren Daten (Stichwort: Informationsfreiheitsgesetze), sondern auch zu Maschinen- bzw. Industriedaten und anderen nicht personenbezogenen Daten, die von Privaten erhoben oder erworben wurden. Ein solches Recht besteht angesichts auch grundrechtlich geschützter Interessen an ggf. sogar exklusiver Eigennutzung bisher nicht, kann aber gesetzlich nach verfassungsgemäßer Abwägung der betroffenen Interessen begründet werden. Es kann nicht zuletzt in Anbetracht der Monopolisierungen oder Oligopolisierungen in wichtigen Teilen der IT-Wirtschaft (Stichwort: Marktversagen) als Gegengewicht bedeutsam sein. ${ }^{120}$ Ohne (ggf. entgeltliche) Zugangsrechte zu Daten und zu ihrer Nutzung im Bereich von Big Data und des Einsatzes von KI können insbesondere Innovationen sowie die Entwicklung alternativer Dienste vergleichbarer Qualität durch andere Unternehmen oder sonstige Akteure erschwert oder unmöglich werden.

118 Dazu s. statt vieler K. H. Fezer, Ein originäres Immaterialgüterrecht sui generis an verhaltensgenerierten Informationsdaten der Bürger, ZD 2017, 99. Zu Diskussionen, in denen auch weitere Schutzvorkehrungen erwogen werden, s. $M$. Fries/M. Scheufen, Märkte für Maschinendaten, MMR 2019, $721 \mathrm{ff} . ;$ T. Riehm, Rechte an Daten - Die Perspektive des Haftungsrechts, VersR 2019, 714, $718 \mathrm{f}$. (T. Hoeren, Datenbesitz statt Dateneigentum, MMR 2019, 5 ff. spricht sich für den Schutz von Datenbesitz - nicht Dateneigentum - aus. Deutlich gegen Eigentumsrechte an Daten L. Determann, Gegen Eigentumsrechte an Daten, ZD 2018, 503.

119 Dazu s. S. Simits/G. Hornung/I. Spiecker gen. Döhmann (Hrsg.), Datenschutzrecht 2019, Einleitung Rn. 27 m. Nachw. in Fn. 61, 62, Rn. 311 m. Nachw. in Fn. 712 - jeweils auch unter Hinweis auf kritische Stimmen; C. J. Haller, Digitale Inhalte als Herausforderung für das BGB, 2019.

120 Zur Diskussion dazu s. auch M. Fries/M. Scheufen (Fn. 118); M. Denga, Gemengelage privaten Datenrechts, NJW 2018, $1371 \mathrm{ff}$, und - auch aus ökonomischer Sicht - W. Kerber, A New (Intellectual) Property Right for Non-Personal Data? An Economic Analysis, GRUR Int. 2016, 989 ff.; M. Ebers, Regulierung, in Ebers et al. (Fn. 5), $\mathbb{3}$, Rn. 91 ff.; H. Schweitzer, Datenzugang in der Datenökonomie: Eckpfeiler einer neuen Informationsordnung, GRUR 2019, $569 \mathrm{ff}$. 
Gesellschaftlich relevante Daten sind heute regelmäßig nicht allein als Produkt des „Datenproduzenten“ zu verstehen. Sie bauen auf den vielen durch die digitale Transformation geschaffenen Möglichkeiten auf, und zwar auf Vorleistungen in Gestalt verfügbarer Hardware, nutzbarer Infrastrukturen, in der Wissenschaft erarbeiteten Knowhows und verfügbarer Dienstleistungen anderer. Dies kann es rechtfertigen, dass der Gesetzgeber unter Verweis auf die Sozialgebundenheit betroffener Daten und das Ziel der Sicherung der Funktionsfähigkeit von Wettbewerb, der Förderung von Innovationen oder der Verfolgung besonderer Gemeinwohlzwecke gewisse Zugangs- und Nutzungsrechte eröffnet.

Eine weitere Problematik betrifft die Frage, wieweit es - ggf. unter gesetzlicher Ausgestaltung der allgemeinen Zugänglichkeit im Hinblick auf die Informationsfreiheit des Art. 5 Abs. 1 Satz 1 GG - Zugangsrechte zu Wissen gibt oder geben sollte. ${ }^{121}$ Gefordert wird vielfach der Ausbau des digitalen Open Access, insbes. in Gestalt des freien Zugangs zu wissenschaftlicher Literatur, ${ }^{122}$ zu Dokumenten sowie auch zu Ergebnissen wissenschaftlicher Forschung und des Rechts zu ihrer (auch digitalen) Auswertung. ${ }^{123}$ Sind die entsprechenden Daten nicht zugänglich, kann vorhandenes Wissen nicht verwendet werden, auch wenn es zur Bewältigung von Zukunftsaufgaben ist. Wichtig sind auch Möglichkeiten/Sicherungen der Interoperabiltät von Software und Netzen und weitere Voraussetzungen für die Nutzung von Gelegenheiten mehrerer zur Zusammenarbeit, dies auch als Mittel der Innovationsermöglichung.

\section{Algorithmische Systeme als Akteure im Rechtsverkehr}

Rechtlich und faktisch besonders delikate Probleme werden im Zusammenhang mit dem verstärkten Einsatz lernender algorithmischer Systeme zu bewältigen sein. Dies insbesondere, soweit sie eigenständig am Rechtsverkehr teilnehmen und Akte mit Rechtsverbindlichkeit vornehmen oder

121 Dazu s. M. Peitz/H. Schweitzer, Ein neuer europäischer Ordnungsrahmen für Datenmärkte? NJW 2018, 275, 279 ff.; A. Wiebe/N. Schur (Fn. ), 467 ff. Auch gibt es mehrere Open-Access-Initiativen, so auch durch das Bundesministerium für Bildung und Forschung.

122 Dies betrifft auch den Zugang rechtswissenschaftlicher Forschung. S. dazu die Beiträge von H. Hamann/D. Hürlimann, J. Rux, I. Vogel und C. Mathieu zu Open Access im Sonderheft 2019 der Zeitschrift Rechtswissenschaft.

$123 \mathrm{Zu}$ Letzterem s. B. Raue, Rechtssicherheit für datengestützte Forschung, ZUM $2019,684 \mathrm{ff}$. 
Rechtspflichten verletzen, die Haftung auslösen können oder aus rechtspolitischer Sicht auslösen sollten oder die sogar strafrechtlich relevant werden. ${ }^{124}$ Darauf hier näher einzugehen, würde den Rahmen sprengen. Stattdessen verweise ich auf zwei besonders detaillierte und innovative Publikationen.

Die eine ist der Aufsatz von Gunther Teubner zu den Fragen, ob es digitale Rechtssubjekte geben sollte, wie deren Verantwortlichkeit bestimmt werden und wie angemessene Instrumente für Haftung ausgestaltet sein könnten. ${ }^{125}$ Er schlägt drei neue Formen eines digitalen Rechtsstatus für autonome Softwareagenten vor: Akteure mit beschränkter Rechtssubjektivität, Mitglieder eines Mensch-Maschine-Verbunds und die Einordnung als Teilelemente eines Risikopools. Der andere Beitrag ist das von Herbert Zech dem 73. Deutschen Juristentag 2020 vorgelegte Gutachten. ${ }^{126}$ Er spricht sich gegen die Einführung einer „e-Person“ als Haftungssubjekt aus, entwickelt aber eine Vielfalt von Konstruktionen, um einerseits durch Verkehrspflichten und Beweislastregeln auf die neuen Herausforderungen zu reagieren und andererseits das Haftungsregime stärker zu differenzieren. Zu letzterem gehört u.a. die Einführung einer Gefährdungshaftung für das Handeln solcher lernender Systeme, die sich durch Robotik und Vernetzung auszeichnen. Ferner schlägt er als Reaktion auf die zunehmende Vernetzung eine haftungsersetzende gesetzliche Unfallversicherung vor.

Solche und die zuvor von mir angesprochenen - die Bandbreite der Probleme nur andeutenden - Erwägungen zeigen, wie stark die digitale Transformation auch in den Bereich des Rechts, darunter auch des Zivilrechts, hineinwirken kann. Im Laufe des Fortschreitens der Digitalisierung und des Aufkommens weiterer mit ihr verbundener lösungsbedürftiger Probleme und neu gefundener Lösungen wird es mit hoher Wahrscheinlichkeit angezeigt sein, sich vermehrt mit transformativem Recht zu beschäftigen.

\section{Als Merkposten: Disruptionen und Transformationen beim Einsatz digitaler Techniken im Recht (Legal Technology/Computational Law)}

Transformationen sind auch deshalb zu erwarten, weil Recht nicht nur ein Instrument ist, um den Einsatz digitaler Techniken zu begleiten und indi-

124 Zur Anwendung von Strafrecht s. K. Gaede, Künstliche Intelligenz - Rechte und Strafen für Roboter? 2019.

125 G. Teubner, Digitale Rechtssubjekte? Zum privatrechtlichen Status autonomer Softwareagenten, AcP 218, 2018, $155 \mathrm{ff}$.

126 H. Zech (Fn. 1 und 76, Entscheidungen). 
vidual- und gemeinwohlverträglich zu gestalten, sondern weil Recht auch selbst die Nutzung digitaler Techniken ermöglicht oder gar erfordern kann. ${ }^{127}$ Der Einsatz digitaler Techniken im Bereich des Rechts wird meist mit dem Begriff der Legal Technology (häufig als Legal Tech abgekürzt) gekennzeichnet. ${ }^{128}$ Andere ziehen den Begriff Computational Law vor ${ }^{129}$ oder kombinieren beide Begriffe in dem Sinne, dass Legal Technology auf der Anwendung von Computational Law beruhe. ${ }^{130}$

Dieses schon sehr aufgefächerte Feld soll hier nicht näher behandelt werden. Ich habe mich mit Legal Tech schon verschiedentlich befasst. Statt einer Wiederholung verweise ich auf zwei Aufsätze von mir ${ }^{131}$ und erwähne im Folgenden nur einzelne Stichworte.

Algorithmische Systeme lassen sich bei der Setzung, Auslegung, Anwendung und Implementierung von Recht nutzen, sei es zur Vorbereitung, zur Rechtfertigung und Kontrolle von Entscheidungen, aber ggf. auch zum automatisierten Entscheiden. Einsetzbar sind sie auch bei der Rechts-

127 Aus der reichhaltigen Literatur zur Veränderung des Rechts durch dessen Digitalisierung s. etwa L. Gschwend/ P. Hettich/M. Müller-Chen/B. Schindler/I. Wildhaber (Hrsg.), Recht im digitalen Zeitalter. Festgabe Schweizer Juristentag 2015 in Sankt Gallen, 2015; G. Buchboltz, Legal Tech. Chancen und Risiken der digitalen Rechtsanwendung, JuS 2017, 955 ff.; V. Boehme-Neßler, Die Macht der Algorithmen und die Ohnmacht des Rechts. Wie die Digitalisierung das Recht relativiert, NJW 2017, 3031 ff.; J. Wagner, Legal Tech und Legal Robots in Unternehmen und den diese beratenden Kanzleien, BB 2017, 898 ff.; C. Ernst, Algorithmische Entscheidungsfindung und personenbezogene Daten, JZ 2017, 1026 ff.; A. Klafki/F. Würkert/T. Winter (Hrsg.), Digitalisierung und Recht, 2017; M. R. Schulz/A. Schunder-Hartung (Hrsg.), Recht 2030. Legal Management in der digitalen Transformation, 2019.

128 S. etwa die Handbücher von S. Breidenbach/F. Glatz (Hrsg.), Rechtshandbuch Legal Tech, 2018; M. Hartung/M. Bues/G. Halbleib (Hrsg.), Legal Tech, Die Digitalisierung des Rechtsmarkts, 2018.

129 So in dem von M. Hildebrandt initiierten Forschungsprojekt „Accounting as a human being in the era of computational law" (COHOBICOL), https:// www.cohubicol.com/contact.

130 S. dazu M. Genesereth in dem Essay: Computational Law. The Cop in the Backseat, Codex X: The Center for Legal Information, Stanford University, 2015. Im Summary heißt es u. a.: "Legal technology based on Computational Law has the potential to dramatically change the legal profession, improving the quality and efficiency of legal services and possibly disrupting the way law firms do business."

131 W. Hoffmann-Riem, Legal Technology - Preconditions, Opportunities, and Risks, Bucerius Law Journal 02/2019, 57 ff.; Ders., Der Umgang mit Wissen bei der digitalen Rechtsanwendung, 145 AöR (2020) i. E. 
beratung oder beim Abbau von Hürden des Zugangs zum Recht. ${ }^{132}$ Betont werden als Vorteile der Nutzung digitaler Techniken insbesondere Arbeitserleichterungen, Möglichkeiten zur Steigerung der Entscheidungsgeschwindigkeit, der Effizienz, der Fähigkeit zur Verarbeitung großer Mengen von gegebenenfalls sehr heterogenen Informationen und verbesserte Möglichkeiten für Folgenabschätzungen. Es bestehen ausgefeilte Möglichkeiten zur Datenkorrelation, wenn auch keine zur Erfassung der im Recht besonders bedeutsamen Kausalbeziehungen. ${ }^{133}$

Allerdings verfügen algorithmische Systeme nach ihrer gegenwärtigen technischen Leistungsmöglichkeiten nicht über manche für das Handeln natürlicher Personen oder bei Interaktionen zwischen ihnen typische, auch bei Akten der Rechtsanwendung wichtige Fähigkeiten. ${ }^{134}$ So fehlt ihnen die Fähigkeit zur Nutzung des für menschliches Handeln wichtigen impliziten Wissens. Defizitär ist die Fähigkeit zur Entwicklung von Kreativität, von Emotionen und zum Einsatz von Intuition, bzw. - wichtig bei Juristen - des Judiz. Bei der für die Auslegung von Normen wichtigen, argumentativ geleiteten Sinndeutung stoßen Algorithmen (jedenfalls bisher) auf Grenzen. ${ }^{135}$ Zumindest beschränkt ist ferner die Fähigkeit, komplexe Abwägungen unter kontextbezogener Justierung der Abwägungskriterien und ihrer Zuordnung vorzunehmen. Probleme bereiten u. a. die Sicherung von Transparenz und Verantwortung sowie die Kontrollierbarkeit der Nutzung insbesondere lernender algorithmischer Systeme.

Dennoch: Digitale Techniken werden angesichts vieler Vorteile in der Zukunft sehr wahrscheinlich stark vermehrt beim Umgang mit Recht eingesetzt werden. Schon jetzt zeichnet sich ab, dass die Digitalisierung nicht nur rechtliche Berufsrollen verändert, sondern auch die Art rechtlicher Regelungen und den praktischen Umgang mit Recht und dabei auch das Regierungs- und Verwaltungshandeln. ${ }^{136}$

Da Legal Tech die Wirkungskraft tragender Elemente eines demokratischen Rechtsstaats verändern kann, ist eine kritische Begleitung auch

132 Eine ausführliche und systematische Übersicht über Tätigkeitsfelder von Legal Tech und entsprechende Begriffe finden sich bei J. Wagner (Fn.127).

133 Zur Begrenzung digitaler Instrumente auf die Erfassung von Korrelationen s. $M$. Hildebrandt (Fn. 40), $37 \mathrm{ff}$.

$134 \mathrm{Zu}$ „technikimmanenten Erkenntnisgrenzen“ s. auch M. Martini (Fn. 5), 58 ff.

$135 \mathrm{Vgl}$. M. Hildebrandt, Law as computation in the era of artificial legal intelligence. Speaking law to the power of statistics, 68 University of Toronto Law Journal 2018, 1.

136 Dazu s. statt vieler T. Wischmeyer, Regierungs- und Verwaltungshandeln durch $\mathrm{KI}$, in: in M. Ebers et al, (Fn. 5), $\$ 20$. 
durch die Rechtswissenschaft geboten, dies nicht nur mit dem Blick auf die Ergebnisse und Durchschaubarkeit sowie Kontrollierbarkeit konkreter Entscheidungsprozesse, sondern auch auf Auswirkungen auf die Leistungskraft und Qualität von Recht und auf die ihm angemessene Methodik. ${ }^{137}$ Auf wichtige Fragen zur Verantwortungs- und Begründungskultur bei digitalen Entscheidungen sind Timo Rademacher und Thomas Wischmeyer in ihren Referaten ${ }^{138}$ schon eingegangen.

\section{Kooperationsbedarfe}

Schließlich möchte ich noch darauf eingehen, dass die digitale Transformation auch im Bereich von Rechtswissenschaft zu neuen Herausforderungen der Kooperation mit anderen Akteuren führt. Ich begrenze mich auf die Notwendigkeit verstärkter interdisziplinärer Kooperation der Setzer, Anwender und Theoretiker des Rechts (1.) sowie auf die der transnationalen Zusammenarbeit der Staaten und anderer Organisationen (2.).

\section{Transformation interdisziplinären Vorgehens - Ferner: Ein neues Anwendungsfeld, die maschinelle Verhaltensforschung}

Der durch digitale Disruptionen und damit verbundene Transformationen ausgelöste evolutionäre oder gar revolutionäre Wandel lässt sich wissenschaftlich nicht angemessen mit einer Haltung disziplinärer Abschottung analysieren. Das gilt auch für die Rechtswissenschaft. Sie muss, soll sie nicht mit einer Art Blindenstock tastend durch die mit der Digitalisierung komplexer gewordene Welt irren, zur transdisziplinären Weitung des Blicks sowie zur trans- und interdisziplinären Kooperation bereit sein.

Infolge der digitalen Transformation erhält die Informatik einen hervorgehobenen, wenn auch nicht monopolartigen Stellenwert. Um dem Rechnung zu tragen, hat beispielsweise an der University of California, Berkeley, eine bestehende interdisziplinäre Abteilung kürzlich einen neuen, auf

$137 \mathrm{Zu}$ letzterem s. die Aufgabenstellung in dem oben (Fn.129) erwähnten Forschungsprojekt COHUBICOL: "The overarching goal is to develop a new hermeneutics for computational law, based on (1) research into the assumptions and (2) the implications of computational law, and on (3) the development of conceptual tools to rethink and reconstruct the Rule of Law in the era of computational law".

138 I.d.B. 
eine erweiterte Aufgabe verweisenden Namen erhalten: Division of Computing, Data Science, and Society (CDSS). Dieser Name wird dahin gehend verstanden, dass auch Rechtswissenschaftler in die Arbeit der Division einbezogen werden. Ein weiteres Beispiel: Die Bucerius Law School wird in Kürze ein „Center für Legal Technology and Data Society“ gründen, das Lehr- und Forschungsinhalte an Überschneidungsstellen von digitalen Techniken und Recht in interdisziplinärer und -nationaler Weise entwickeln soll. Auch andere Hochschulen arbeiten an „Data Literacy Education"-Projekten zwecks Vermittlung von zukunftsorientierten Kompetenzen und zur Förderung entsprechender Forschung, so die Leuphana-Universität Lüneburg: „Data-driven x“ bzw. „DATA-x“. Die Bemühungen um Interdisziplinarität dürfen sich aber nicht auf solche Einzelinitiativen begrenzen. Die Digitalisierung bedeutet eine Herausforderung für die Rechtspraxis, Rechtslehre und Rechtswissenschaft allgemein.

Eine spezifische aktuelle transdisziplinäre Herausforderung von Forschung möchte ich an einem Beispielsfeld illustrieren, dem Umgang mit KI im Bereich selbstlernender algorithmischer Systeme. In solchen Systemen wird die digitale Automatisierung von Entscheidungen um das Element der technologischen - also nicht menschlichen - Autonomie ergänzt. ${ }^{139}$ Das System ist nicht allein auf Eingaben der Programmierer angewiesen, sondern verlässt sich ergänzend und verändernd auf eigene Wahrnehmungen und kann dabei auch zur Änderung seines eigenen Steuerungsalgorithmus befähigt sein (s. o. I.2). Zu den möglichen Anwendungsfeldern gehören die Beurteilung der Kreditwürdigkeit einer Person, die Abwicklung finanzieller Transaktionen, die Vorhersage des Rückfallrisikos von Straftätern, Online-Dating, dynamische Preisgestaltungen, automatisiertes Kfz-Fahren, die Beeinflussung von Verhalten im Konsumbereich oder bei politischen Wahlen sowie auch der intelligente Einsatz von Drohnen zur Überwachung und zur Kriegsführung. Diese und weitere Einsatzmöglichkeiten können massiv auf individuelles und kollektives Verhalten einwirken - sei es mit positiv, sei es mit negativ zu bewertenden Folgen. Die dabei zu bearbeitenden Probleme sind inhaltlich häufig sehr komplex, so dass trans- und interdisziplinäre Kooperation ein sinnvoller, wenn nicht gar zwingender Ansatz zur Bewältigung der Komplexität ist.

Die Fähigkeit zu autonomem Handeln wird traditionell in erster Linie bei Menschen verortet und die Möglichkeit dazu rechtlich geschützt. Jetzt muss verarbeitet werden, dass lernende algorithmische Systeme über Autonomie beim Lösen von Aufgaben verfügen. Über die daraus resultierenden 
Folgen gibt es auch in der Rechtswissenschaft viele Diskussionen, so - wie schon erwähnt - etwa zu Fragen nach der (Teil-)Rechtsfähigkeit von lernenden Systemen ${ }^{140}$ oder der Zuordnung von Haftung. ${ }^{141}$

Ich möchte hier darauf nicht weiter eingehen, wohl aber das spezifische Problem erneut ansprechen, dass die Handhabung von Autonomie durch Maschinen für Menschen nicht oder doch weitgehend nicht transparent und deshalb auch nicht oder nur sehr schwer aufklärbar und rechtlich kontrollierbar ist. ${ }^{142}$ Das ist in einem Rechtsstaat nicht hinnehmbar, es sei denn, es gäbe Möglichkeiten zur Sicherung hinreichender Durchschaubarkeit lernender algorithmischer Systeme: Wie kommen sie im konkreten Fall zu Problemlösungen und weiter: Welche Informationen sind unabdingbar, um eine rechtsstaatliche Kontrolle zu ermöglichen?

Um die Spezifika autonomen Handelns algorithmischer Systeme erfassen zu können, hat eine Gruppe amerikanischer Wissenschaftler die Bildung einer neuen wissenschaftlichen Disziplin gefordert: Die maschinelle Verhaltensforschung (Forschung über "machine behaviour"143). Sie verweisen insbesondere darauf, dass komplexe (lernende) KI-Agenten nicht länger allein aus ihrem internen Bauplan heraus verstanden werden können, sondern nur im Zusammenspiel von Maschine und Umwelt. ${ }^{144}$ Hier hat Wissenschaft einen Aufklärungsauftrag. Maschinelle Verhaltensforschung, deren Ergebnisse auch für die Rechtswissenschaft und -praxis verwendbar sein sollen, erfordert in methodischer und personeller Hinsicht eine Erweiterung trans- und interdisziplinären Vorgehens. Unverzichtbar sind die verstärkte Einbeziehung von Informatikexperten, die Entwicklung von Wegen wechselseitiger Verständigung mit Juristen - auch unter Bildung geeigneter Brücken- und Schlüsselbegriffe -, der Abgleich der „Denkstile“ und genutzten Narrative sowie die Sicherung der Kompatibilität methodischer Vorgehensweisen.

140 Dazu s. etwa G. Teubner (Fn.125).

141 Dazu s. etwa H. Zech (Fn. 76).

142 Dazu s. etwa T. Wischmeyer (Fn. 74); Ders. i. d. B. Zur Relativierung der hohen Bedeutung von Transparenz zur Sicherung von Zurechenbarkeit und Verantwortung s. J. A. Kroll/J. Huey/S. Barocas/E. W. Felten/J. R. Reidenberg/D. G. Robinson $/ H$. Yu, Accountable Algorithms, University of Pennsylvania Law Review, 165, 2017, 633.

143 I. Rahwan et al., Machine behaviour, nature 2019, 477, 480.

144 So die Umschreibung von B. Fateh-Moghadam (Fn. 91), 869. 


\section{Notwendigkeit der Transformation der internationalen Kooperation}

Schließlich sei als Merkposten erwähnt, dass der Einsatz von Recht auch darauf abgestimmt sein muss, dass digitale Techniken nicht nur weltweit eingesetzt werden, sondern dass viele digitalen Dienste trans- und international stark vernetzt sind. Die digitale Transformation muss sich daher auch auf die Entwicklung von Recht auswirken, das es erlaubt, Regelungsaufgaben unter Bedingungen der Digitalisierung angemessen wahrzunehmen.

Vor allem angesichts der territorialen Entgrenzungen und Vernetzungen reichen nationale Bemühungen unter Einschluss nationaler Rechtsregeln vielfach nicht zur Problembewältigung aus. Auf die globale bzw. transnationale Dimension der digitalen Transformation wurde schon mehrfach verwiesen. Die Problembewältigung darf - wie erwähnt (s. o. VII) - angesichts des Risikos einseitiger Interessenverwirklichung nicht allein der Selbstregelung/-regulierung der machtvollen international tätigen Unternehmen überlassen werden. Zumindest bedürfen die von diesen geschaffenen Regeln der hoheitlich verantworteten regulativen Umhegung (vgl. o. VI). Nationale Normgeber können zwar auf den Einsatz digitaler Techniken in ihren jeweiligen Hoheitsgebieten einwirken. Sie sind relativ machtlos gegenüber Ausweichstrategien transnational tätiger Unternehmen. Zu solchen Strategien gehören z. B. die gezielte Wahl der Standorte der technischen Systeme, oder des Sitzes des Unternehmens oder die Verlagerung von Aktivitäten in Staaten mit geringeren rechtlichen Anforderungen. ${ }^{145}$

Anzustreben ist die Schaffung transnational und global wirksamer, im Mehrebenensystem internationaler, supranationaler und nationaler rechtlicher Regelungsstrukturen. ${ }^{146}$ Die normativen Vorgaben und Vollzugsvorkehrungen sollten möglichst auch in entsprechenden trans-/internationalen Übereinkommen verankert sein. $\mathrm{Zu}$ erarbeiten sind insofern neue Konzepte, Vereinbarungen und Einrichtungen einer transnationalen ITGovernance. ${ }^{147}$ Die Bemühungen sollten auch auf Kooperation hoheitlicher Akteure mit den betroffenen Stakeholdern ausgerichtet sein, nicht

145 Dazu s. P. Nemitz, Constitutional Democracy and Technology in the Age of Artificial Intelligence, em: Philosophical Transactions of the Royal Society, A 376, 2018, http://dx.doi.org/10.1098/rsta.2018.0089.

146 M. Oermann (Fn. 4), 315.

$147 \mathrm{Zu}$ dem Themenfeld Governance s. die Nachw. in Fn. 47. Angaben zu internationalen Initiativen zur Regelung von KI und Robotik finden sich bei M. Ebers, (Fn.120), \$3, Rn. 147 ff., 170 ff. 
nur mit den Verbänden und Unternehmen der digitalen Wirtschaft, sondern ebenfalls mit Nichtregierungsorganisationen (NGOs) und anderen Vertretern zivilgesellschaftlicher Interessen. ${ }^{148}$

Für die folgenreiche Verwirklichung eines solchen Anliegens gibt es allerdings bisher nur wenig Anhaltspunkte. Den Interessen der machtvollen IT-Unternehmen - insbesondere der US-amerikanischen und chinesischen Unternehmen - sowie der Regierungen ihrer „Heimat"-Staaten entspricht dies offenbar nicht oder nur begrenzt. Die im Zuge der digitalen Transformation geschaffenen Marktstrukturen und die über sie ermöglichten Machtasymmetrien sind jedenfalls bisher eine schwer zu überwindende Barriere zur kooperativen transnationalen Verständigung.

Insoweit bleibt aber immerhin der Weg, den Wunsch der Unternehmen auf den ökonomisch lukrativen Zugang zu dem relativ großen europäischen Markt und zu den Märkten einzelner größerer Volkswirtschaften zu nutzen und den Zugang zumindest von der Beachtung regulativer Vorkehrungen abhängig zu machen, die hinreichenden Rechts- und Interessenschutz der Nutzer sowie die Wahrung rechts- und demokratiestaatlicher Grundsätze sichern. Im Interesse der transnational tätigen, global wichtige Leistungen erbringenden Unternehmen liegt es nicht, auf unterschiedlichen Märkten je unterschiedliche Vorgaben beachten zu müssen. Dies kann eventuell als Anreiz für kooperative Verständigung genutzt werden sicher ist der Erfolg aber nicht.

\section{Ausblick}

Die Perspektive auf die Disruption und Transformation ist für die Analyse der gegenwärtigen Entwicklung der Digitalisierung und ihrer Folgen hilfreich. Die relative Unvorhersehbarkeit der weiteren Entwicklung und der Verwirklichung von Chancen und Risiken und der diese begleitenden weiteren transformativen Veränderungen sollten Anlass sein, fortlaufend nach angemessenen Reaktionen in den verschiedenen Bereichen der Gesellschaft zu suchen. Recht ist einer von mehreren Ansatzpunkten zur Gestaltung der Gegenwart und Zukunft. Ebenso wichtig sind wirtschaftliche, kulturelle und soziale Rahmenbedingungen sowie gesellschaftliche Nor-

148 Vgl. W. Hoffmann-Riem, (Fn. 48), 691-693 m. w. Hinw. Allgemein zum Zusammenspiel unterschiedlicher Akteure unter eingehender des Handelns von NGOs s. J. von Bernstorff, Die Rolle nicht-staatlicher Akteure bei der Entwicklung und Implementierung des Völker- und Europarechts, VVDStRL 79, 2020, $381 \mathrm{ff}$. 
men, insbesondere ethische und moralische. Nichtrechtliche und rechtliche Normen beeinflussen sich allerdings wechselseitig. ${ }^{149}$

Rechtswissenschaft kann und sollte die Gelegenheit nutzen, die transformativen Potentiale der Digitalisierung aus rechtlicher Sicht zu analysieren und die eigenen Möglichkeiten der Mithilfe bei der Zukunftsgestaltung zu nutzen und weiterzuentwickeln. Dafür wird es wichtig sein, die transdisziplinäre Kompetenz und die transnationale Orientierung auszuweiten.

In welchen Hinsichten die durch die digitale Disruption gegebenen Anstöße zu grundlegenden Änderungen in der Rechtsordnung führen, ist einstweilen offen. Die gesamtgesellschaftlich folgenreiche digitale Transformation hat jedenfalls das Potential, auch erhebliche Transformationen im Recht auszulösen, die - hoffentlich - helfen, Möglichkeiten zur Verbesserung von Lebensverhältnissen sowie zur Vermeidung bzw. Bewältigung von Risiken auszubauen. Unabdingbar sollten dabei Vorkehrungen sein, die auch angesichts der transformativen Veränderungen die Leistungsfähigkeit rechtsstaatlicher Grundsätze und die demokratische Legitimation des Handelns sichern.

149 Dass dies sehr produktiv sein kann, zeigt eindrucksvoll das Gutachten der Datenethikkommission, 2019. 